\title{
Variation of different components of Jupiter's auroral emission
}

\author{
J. D. Nichols, ${ }^{1,2}$ J. T. Clarke, ${ }^{1}$ J. C. Gérard, ${ }^{3}$ D. Grodent, ${ }^{3}$ and K. C. Hansen ${ }^{4}$
}

Received 7 January 2009; revised 2 March 2009; accepted 11 March 2009; published 16 June 2009.

[1] The Hubble Space Telescope (HST) data set obtained over two campaigns in 2007 is used to determine the long-term variability of the different components of Jupiter's auroras. Three regions on the planet's disc are defined: the main oval, the low-latitude auroras, and the high-latitude auroras. The UV auroral power emitted from these regions is extracted and compared to estimated solar wind conditions projected to Jupiter's orbit from Earth. In the first campaign the emitted power originated mainly from the main oval and the high-latitude regions, and in the second campaign the high-latitude and main oval auroras were dimmer and less variable, while the low-latitude region exhibited bright, patchy emission. We show that, apart from during specific enhancement events, the power emitted from the poleward auroras is generally uncorrelated with that of the main oval. The exception events are dawn storms and compression region enhancements. It is shown that the former events, typically associated with intense dawnside main oval auroras, also result in the brightening of the high-latitude auroras. The latter events associated with compression regions exhibit a particular auroral morphology; that is, where it is narrow and well defined, the main oval is bright and located $\sim 1^{\circ}$ poleward of its previous location, and elsewhere it is faint. Instead there is bright emission in the poleward region in the postnoon sector where distinct, bright, sometimes multiple arcs form.

Citation: Nichols, J. D., J. T. Clarke, J. C. Gérard, D. Grodent, and K. C. Hansen (2009), Variation of different components of Jupiter's auroral emission, J. Geophys. Res., 114, A06210, doi:10.1029/2009JA014051.

\section{Introduction}

[2] Jupiter's ultraviolet (UV) auroras are generally divided into three components. With increasing latitude these are the moon footprints, the main auroral oval emission (or simply main emission), and the polar auroras. The satellite footprints are magnetically linked to the Galilean satellites, and the brightest usually maps to the volcanic satellite Io, which orbits deep within the magnetosphere at $\sim 5.9 \mathrm{R}_{\mathrm{J}}$. The Io footprint has been shown to be a complex entity, consisting of multiple spots whose brightness and location have previously been shown to be determined by the location of Io in its orbit and the surrounding plasma torus [Gérard et al., 2006; Serio and Clarke, 2008; Bonfond et al., 2008]. We therefore do not consider the variation in brightness of this auroral component here.

[3] The main auroral oval is the brightest and most stable component of Jupiter's auroras, varying on the timescale of tens of hours, and maps to the middle magnetosphere

\footnotetext{
${ }^{1}$ Center for Space Physics, Boston University, Boston, Massachusetts, USA.

${ }^{2}$ Department of Physics and Astronomy, University of Leicester, Leicester, UK.

${ }^{3}$ Laboratory for Planetary and Atmospheric Physics, Institut d'Astrophysique et de Géophysique, Université de Liège, Liege, Belgium.

${ }^{4}$ Department of Atmospheric, Oceanic and Space Sciences, University of Michigan, Ann Arbor, Michigan, USA.
}

Copyright 2009 by the American Geophysical Union. 0148-0227/09/2009JA014051
[Grodent et al., 2003a; Clarke et al., 2002, 2004]. It is thought to be associated with the breakdown of corotation of iogenic plasma beyond $\sim 20 \mathrm{R}_{\mathrm{J}}$ [Cowley and Bunce, 2001; Hill, 2001; Khurana, 2001; Nichols and Cowley, 2004]. The main oval is generally observed to be narrow and cohesive between System-III (SIII) longitudes $\sim 160-$ $240^{\circ}$, and diffuse and disordered at smaller longitudes, a region that appears to contain a local magnetic anomaly [Grodent et al., 2008a] (note that the SIII prime meridian lies on the opposite side of the planet to the main auroral oval, such that "smaller" longitudes generally refer to those toward the right, and vice versa, as observed from Hubble Space Telescope (HST)). We should note, however, that a significant observational bias exists, such that meridians are generally observed at similar local times. This leads to ambiguity between local time and longitudinal effects, and work is currently underway to resolve this effect. In the mean time, we generally refer to longitudes here, but the local time ambiguity should be borne in mind. While the morphology of Jupiter's main oval is relatively stable on the timescale of one HST visit (roughly an hour), it has been observed to vary significantly on longer timescales [Grodent et al., 2003a, 2008b]. For example, the main oval occasionally exhibits intense "dawn storms", in which the dawn sector brightens and expands considerably [e.g., Gustin et al., 2006], a phenomenon which may be related to spots that appear just poleward of the main oval on the dawnside with an apparent periodicity of 2-3 days [Radioti et al., 2008]. Simple theoretical arguments suggest that the brightness of 
the main auroral oval should be anticorrelated with the solar wind dynamic pressure [Southwood and Kivelson, 2001; Cowley and Bunce, 2001, 2003a, 2003b]. This arises since under magnetospheric compression the angular velocity of the equatorial plasma increases because of conservation of angular momentum. The corresponding corotation-enforcement currents, the upward field-aligned component of which is responsible for the main oval auroras, will then be decreased, and vice versa. In reality, as discussed by Cowley et al. [2007], the response to solar wind conditions may be much more complex than this simple picture, and this is discussed in greater detail in section 7 .

[4] Unlike the main oval emission, the polar auroras are highly variable on all observed timescales, i.e., down to tens of seconds. Grodent et al. [2003b] identified various subcomponents of the polar auroras, namely the "active region", the "swirl region" and the "dark polar region". The active region lies toward noon, regularly exhibits bright but transient spots, and has been observed to "flare" from a few $\mathrm{kR}$ (where 1 Rayleigh represents a photon source flux of $10^{6} \mathrm{~cm}^{-2} \mathrm{~s}^{-1}$ radiating into $4 \pi$ steradians) to $\sim 10 \mathrm{MR}$ over a few minutes [Waite et al., 2001]. The swirl region occupies the most poleward territory, and exhibits fainter, patchy and highly variable emission generally of order $\sim 10 \mathrm{kR}$ but sometimes reaching a few hundred $\mathrm{kR}$. The dark polar region, almost devoid of emission, lies between the main oval and polar auroras on the dawn, but sometimes extends round to the dusk [Nichols et al., 2007]. Transient "inner ovals" a few degrees poleward of the main oval have also been reported [Ballester et al., 1996; Pallier and Prangé, 2001; Nichols et al., 2007], similar to those predicted by the models of Cowley et al. [2005, 2007]. It has been suggested that at least a component of the polar auroras is related to the solar wind interaction at the dayside boundary between open and closed field lines [Clarke et al., 1998; Pallier and Prangé, 2001; Waite et al., 2001; Grodent et al., 2003b], and may exhibit a direct relation to the conditions in the interplanetary medium. This idea was explored theoretically by Bunce et al. [2004], who showed that pulsed dayside reconnection during periods of strong solar wind interaction could excite adjacent high-latitude regions of UV and X-ray emission, as observed by Elsner et al. [2005]. Cowley et al. [2007] also showed that highlatitude auroras should be generated at the open-closed field line boundary because of the flow shear between open and closed flux. Under compression this shear is enhanced because of the increase in angular velocity of equatorial plasma as discussed above, such that this emission will brighten, and vice versa.

[5] The long-term behavior of the different components of Jupiter's auroras is not well understood, since the nature of previous HST observations is such that high-sensitivity images have been obtained over relatively short periods of time, generally a few days. Equally problematic is the fact that simultaneous upstream solar wind data are limited to rare flybys. Until 2007 the most recent of these was the Cassini flyby of December 2000 to January 2001, which led to a number of studies showing that the total UV and radio emitted power increased by factors of 2-4 during compression regions, i.e., intervals of enhanced interplanetary magnetic field (IMF) magnitude and dynamic pressure associated with corotating interaction regions (CIRs)
[Gurnett et al., 2002; Pryor et al., 2005; Nichols et al., 2007]. The spatially resolved HST images of Jupiter's auroras obtained during this interval were examined in conjunction with Cassini data by Nichols et al. [2007]. They showed that of the 177 HST images obtained all but two were taken during rarefaction regions of low IMF magnitude and dynamic pressure. The two images obtained during a compression region both had high central meridian longitude (CML) values, such that the view of the auroras was somewhat limited. In addition, the Cassini spacecraft was repeatedly moving in and out of the bow shock and magnetopause, rendering genuine solar wind data patchy and the exact timing of the compression region onset was uncertain. However, on the basis of these two images it was shown that the all components of the auroras were brighter than usual during the compression event. The main oval was bright along its entire length, and latitudinally expanded poleward by $\sim 1-2^{\circ}$. In the postnoon sector the expanded oval merged with bright poleward forms, which were taken to be enhanced active region auroras. The rest of the poleward region was filled with patchy swirl region-type emission. However, given the paucity of the solar wind data and small number of HST images taken during the compression region, it was not possible to determine the repeatability of these phenomena or the precise solar wind trigger of the brightened auroras.

[6] In 2007 a major campaign of observation was undertaken with HST in which Jupiter was observed once a day during two month-long campaigns. The first coincided with the New Horizons flyby in February, while the second took place near opposition in June. This campaign represents the first opportunity to determine the behavior of the different components of the auroras over a long period of time. The overall variation of total emitted power, along with the key events of the campaign has been discussed by Clarke et al. [2009]. In particular they compared the total emitted UV power with the estimated solar wind dynamic pressure and bulk velocity, propagated from data taken at Earth using a 1D magnetohydrodynamic (MHD) model [Zieger and Hansen, 2008]. They showed that, with some uncertainty in the timing of the propagated data, the total emitted power generally increased in response to the forward shocks associated with CIRs, i.e., events in which the bulk velocity and dynamic pressure increased rapidly. There appeared to be no increase in power associated with the reverse shocks in which the dynamic pressure rapidly decreased, in apparent contradiction with the above theoretical expectations. The occurrence of dawn storms was shown to be independent of the propagated solar wind conditions. However, Clarke et al. [2009] did not discuss which auroral components were responsible for the changes in auroral power, nor did they consider how the different components behave with respect to each other. In this paper we focus on the individual behaviors of the main oval, high-latitude, and low-latitude auroras in response to the conditions in the interplanetary medium.

\section{Data}

\subsection{HST Images}

[7] HST images of Jupiter's auroras were taken over days of year (DOY) 51-70 and 131-162 of 2007 using the Advanced Camera for Surveys (ACS) Solar Blind Channel 
(SBC). The ACS/SBC detector is a $1024 \times 1024$ MultiAnode Microchannel Array, with an average resolution of $\sim 0.032$ arcsec pixel ${ }^{-1}$, such that the field of view is $35 \times$ $31 \operatorname{arcsec}^{2}$. The telescope was oriented such that approximately a quarter of the field of view was filled with the planet's disk, to avoid saturating the detector. Images were obtained using the F115LP and F125LP longpass filters, the former of which admits $\mathrm{H}_{2}$ Lyman and Werner bands and $\mathrm{H}$ Lyman- $\alpha$ emission, while the latter mostly excludes the $\mathrm{H}$ Lyman- $\alpha$ band. Each visit consisted of 15 images spanning $\sim 1 \mathrm{~h}$. Most (907) images were of the northern auroras, while the remainder (580) were of the southern. The exposure times were mostly $100 \mathrm{~s}$, for which the blurring at the CML of any corotating features is $\sim 1^{\circ}$, but some short-duration $30 \mathrm{~s}$ images were also obtained. The images were reduced using a pipeline developed at Boston University which utilizes the latest dark count, flat field and geometric distortion correction reference files available from the Space Science Telescope Institute. As part of the reduction the images were rotated such that the north pole was oriented toward the top and scaled to a standard distance of 4.2 AU using ephemerides obtained from the NASA Navigation and Ancillary Information Facility SPICE system [Acton, 1996]. The units were converted from counts to $\mathrm{kR}$ of $\mathrm{H}_{2}$ and Lyman- $\alpha$ emission using the conversion factors $1 \mathrm{kR}=2.103 \times 10^{-3}, 1.473 \times 10^{-3}$ counts for the F115LP and F125LP filters, respectively, values computed using the Gérard et al. [2002] synthetic spectrum. The center pixel of the planet was found by fitting the planet's limb to a simulated limb profile that takes into account the planet's obliquity and terminator. The accuracy of the center pixel location is estimated to be 4 pixels in the horizontal direction and 3 pixels in the vertical direction. All images are publicly available at $\mathrm{http}: / / \mathrm{www} \cdot b u . e d u / c s p /$ PASS/main.html.

[8] Planetocentric projections were created from the reduced images which allow the simulation of the view as if looking from any desired viewpoint about the planet. The accuracy of the projection decreases toward the limb, because of the increasing obliquity of the planet's surface to the observer. Details of the accuracy of the projection can be found in the work of Grodent et al. [2003a]. Finally, the disk of reflected sunlight was subtracted by fitting Minnaert functions separately to the dawn and dusk halves of the disk [Vincent et al., 2000], and applying latitudinal intensity patterns obtained for each month by summing the images in which the auroras were on the nightside of the planet.

\subsection{Interplanetary Data}

[9] In common with Clarke et al. [2009] we use the estimated conditions in the interplanetary medium propagated to Jupiter from Earth using the 1D MHD model of Zieger and Hansen [2008]. The model input is data taken by the Advanced Composition Explorer (ACE) spacecraft located near $1 \mathrm{AU}$. The model has been tested extensively by Zieger and Hansen [2008], who showed that the accuracy is highest when the heliosphere is well ordered during solar minimum, and when the Sun, ACE and target are aligned. The year 2007 was near solar minimum, and the second campaign occurred when Jupiter was near opposition, such that for this campaign both conditions were met. The one standard deviation uncertainty in the timing of arrival of solar wind events at Jupiter is estimated to be $\pm 15 \mathrm{~h}$ in this case [Clarke et al., 2009]. The first campaign occurred 100 days before opposition, such that the timing uncertainty is $\pm 24 \mathrm{~h}$, higher than for the second campaign. In the case of the first campaign, the New Horizons spacecraft was in the solar wind until day 56, and observed an interplanetary shock on day 53 [Elliott, 2007]. Clarke et al. [2009] used this information to calibrate the MHD projection timing, and applied a constant shift of +2.1 days to the model results over this campaign. In this study we show the results of both the shifted and unshifted model, since it is unclear as to whether the shift is applicable over the whole campaign interval.

[10] The 1D MHD model assumes spherical symmetry of the solar wind and corona, and is not able to model the radial component of the magnetic field $B_{\mathrm{R}}$, because of the condition div $\mathbf{B}=0$. Note that we use here the heliocentric spherical RTN coordinate system, in which the R direction is radial, $\mathrm{N}$ is parallel to the Sun's spin axis and T forms the right-handed set, roughly in the direction of planetary motion. The parameters modeled are, in order of decreasing accuracy, the solar wind bulk velocity $v_{\mathrm{sw}}$, the magnetic field magnitude $|B|$, the solar wind plasma density $\rho$, the azimuthal magnetic field component $B_{\mathrm{T}}$, the plasma temperature $T$, and finally the north-south component of the field $B_{\mathrm{N}}$, which is essentially a null prediction. The parameters of interest in determining the response of the auroras to the conditions in the interplanetary medium are the solar wind dynamic pressure given by

$$
p_{\mathrm{dyn}}=\rho v_{\mathrm{sw}}^{2},
$$

and the dayside reconnection voltage $\phi$, which is equal to the rate of open flux production at the dayside magnetopause. The reconnection voltage, estimated for example by the Nichols et al. [2006] algorithm given by $\phi=v_{\mathrm{sw}} B_{\perp} L_{\mathrm{o}}$ $\cos ^{4}\left(\theta_{\mathrm{IMF}} / 2\right)$, is dependent on the component of the IMF perpendicular to the radial direction $B_{\perp}$, the width $L_{\circ}$ of the channel in the solar wind that reconnects at the magnetopause for exactly northward IMF, and the IMF clock angle $\theta_{\mathrm{IMF}}$, i.e., the angle measured clockwise between the IMF and the planet's magnetic dipole axis as projected into the T-N plane. However, $B_{\mathrm{N}}$ is not accurately computed by the MHD model, such that the values of $B_{\perp}$ and $\theta_{\mathrm{IMF}}$ remain unknown. In light of this uncertainty, in the analysis that follows we do not explicitly compute the reconnection voltage and consider only $|B|$ and the sense of $B_{\mathrm{T}}$.

\section{Analysis}

\subsection{Auroral Regions}

[11] Jupiter's internal field is significantly nondipolar, such that the northern auroras are displaced further from the spin pole than the southern and the view of the northern auroras from Earth is thus more complete. For this reason, in this paper we concentrate on the northern auroras, and for consistency we consider only images taken with the F125LP filter. We consider three different auroral components individually, based roughly on the three components discussed in section 1. Specifically, we consider the highlatitude (HL) emission, the main oval (MO) emission and a 


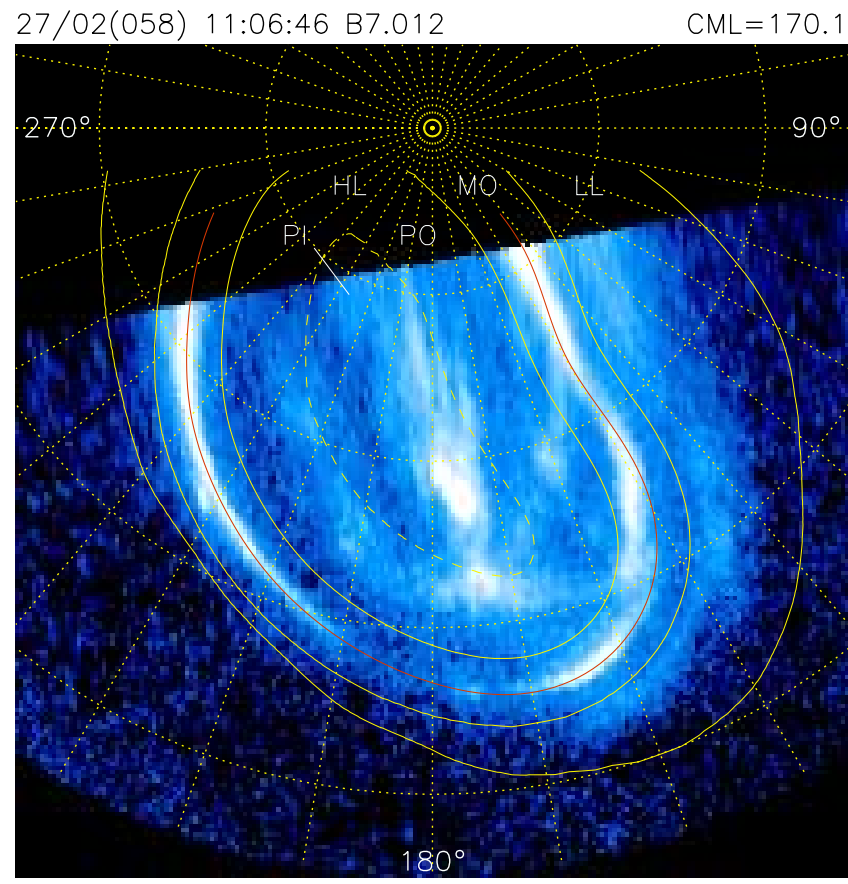

Figure 1. A typical projected Hubble Space Telescope (HST) image of Jupiter's auroras, obtained on 27 February 2007. The simulated view is from above the north pole, and the image is displayed with a log color scale saturated at $500 \mathrm{kR}$. The red line shows the reference main oval as given by the locations in Table 1 . The solid yellow lines show the boundaries between the high-latitude region, the main oval, and the low-latitude emission. The dashed yellow line indicates the boundary between the polar inner and polar outer regions. The yellow points indicate a $10^{\circ} \times 10^{\circ}$ planetocentric latitude-SIII longitude grid. The image is oriented such that SIII longitude $180^{\circ}$ is directed toward the bottom.

third component not mentioned in section 1, i.e., the lowlatitude (LL) non-Io emission.

[12] In Figure 1 we show a typical HST image, projected such that the simulated viewpoint is from above the north pole, and with the boundaries between these auroral regions overlaid in solid yellow lines. A movie of all the projections in a similar format is available in the auxiliary material (Movie S1). ${ }^{1}$ We have defined the main oval region to be a strip of width $4^{\circ}$ orthogonal to and centered on the statistical location of the main oval, obtained when all the images are overlaid in a manner similar to Grodent et al. [2003a]. The coordinates of this reference oval are shown in Table 1. These coordinates are different to those derived by Grodent et al. [2003a], since, as documented by Grodent et al. [2008b], the main oval has shifted in location since 2001, possibly because of a change in the middle magnetosphere current sheet. The chosen width of the strip was somewhat arbitrary, and is a compromise between covering the full range of motion of the main oval, and not including too much unrelated polar emission. The high-latitude emission

${ }^{1}$ Auxiliary materials are available in the HTML. doi:10.1029/ 2009JA014051. was then defined as any aurora poleward of the main oval strip, and the low-latitude emission was defined as being any aurora between the main oval region and a line $1.5^{\circ}$ poleward of the Io footprint contour defined by Grodent et al. [2008a, Table 1]. The low-latitude emission is thus expected to map to the equatorial plane between the vicinity of Io's orbit and the main oval region, i.e., to the inner region of the middle magnetosphere $\sim 6-20 \mathrm{R}_{\mathrm{J}}$. The main oval region then maps to the middle magnetosphere in the region of $\sim 20-40 \mathrm{R}_{\mathrm{J}}$ [Nichols and Cowley, 2004] and the polar region is expected to map to the outer magnetosphere and open flux regions.

[13] In order to further characterize the variable highlatitude region we have also divided this region into two subcomponents, the polar inner (PI) and polar outer (PO) regions. The boundary between the two, shown by the dashed yellow line in Figure 1, was chosen to lie $7^{\circ}$ poleward of the statistical main oval. Again, this location is somewhat arbitrary, and was chosen to enclose as much of the very high latitude swirl region auroras as possible, whilst excluding the bright arcs that are occasionally observed poleward of the main oval (discussed further in section 4).

[14] It is important to note that the boundaries imposed here are artificial and it is inevitable that in the $\sim 1000$ images some bright emission will straddle the boundary, although we have chosen the locations to minimize this occurrence. In addition, if from one visit to the next an auroral feature such as an arc of bright emission along the reference main oval dims and is replaced by a similar form, say a few degrees poleward, this can be interpreted as being either the same feature that has moved, or the manifestation of two independent events. This ambiguity can only truly be removed with comprehensive simultaneous in situ measurements. Thus, in the absence of in situ evidence that specifically links two arguably independent auroral forms,

Table 1. SIII Coordinates of the Reference Main Oval Derived From the Average of All the Images

\begin{tabular}{cc}
\hline SIII Longitude $(\mathrm{Deg})$ & Latitude $(\mathrm{Deg})$ \\
\hline 277.1 & 88.6 \\
282.8 & 86.1 \\
273.5 & 82.9 \\
260.9 & 78.9 \\
252.0 & 76.7 \\
240.0 & 73.7 \\
230.0 & 70.8 \\
220.0 & 67.5 \\
210.0 & 64.2 \\
200.0 & 61.4 \\
190.0 & 58.8 \\
180.0 & 56.7 \\
170.3 & 55.6 \\
164.3 & 56.0 \\
159.4 & 57.2 \\
153.8 & 59.9 \\
150.0 & 63.6 \\
148.9 & 68.7 \\
149.7 & 74.2 \\
143.1 & 80.8 \\
143.1 & 85.6 \\
185.7 & 89.1 \\
\hline
\end{tabular}



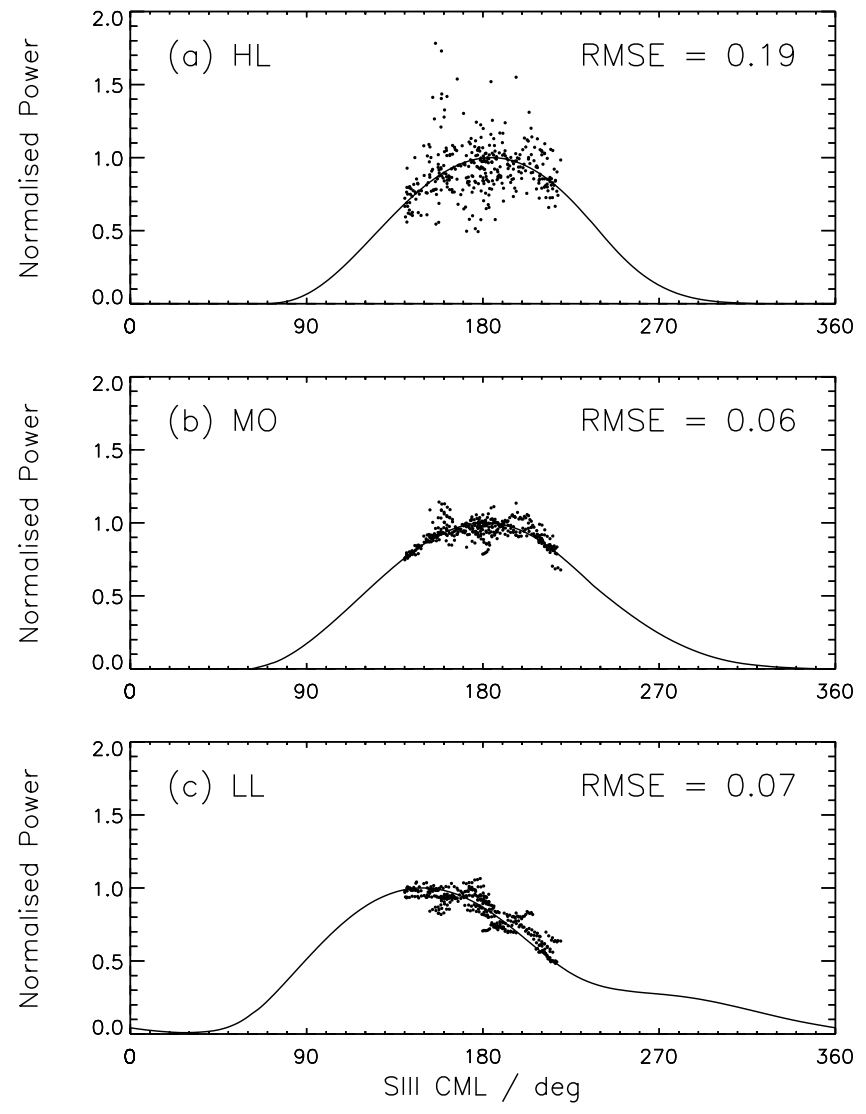

Figure 2. Simulated normalized flux versus central meridian longitude (CML) profiles for each auroral region shown in Figure 1, i.e., (a) the high-latitude region, (b) the main oval, and (c) the low-latitude region. Also shown are the measured power values, scaled for each visit to the single normalized simulated curve. Values of the root-meansquare error between the measured values and the curve are shown for each region.

in this paper we adopt the conservative approach and consider them to be independent features. We can therefore define one set of boundaries for all images, and show how the emission varies with respect to fixed regions, without changing our definition of what constitutes the "main oval" for each image. We thus note that, for example, bright poleward arcs or equatorward patches appearing while the main oval region is dim may in fact represent shifted main oval emission, but we do not explicitly make that assumption here. However, in order to test whether our results would change significantly with the employment of a dynamic definition of the main oval, we have also produced results with main oval region definitions tailored for each image. For longitude bins along the narrow section of the main auroral oval, we determined the difference in degrees of arc of the maximum brightness of the main oval from the reference oval in order to determine the mean deviation from the reference oval. We then defined scaled ovals on the basis of these mean deviations and produced main oval strips and high-latitude region definitions as before. We note here that the difference between the results obtained with the dynamic definition of the main oval and those obtained with the fixed oval region is comparable to the uncertainty in the CML correction method discussed in section 3.2, such that we are confident the fixed oval captures the behavior of the main oval sufficiently well.

\subsection{Emitted UV Power}

[15] A basic parameter that describes the auroral activity at a given time is the total emitted UV power, the variation of which during the 2007 HST campaigns has been discussed by Clarke et al. [2009]. Here we consider the power emitted from the individual auroral components defined in section 3.1 , i.e., $P_{\mathrm{HL}}, P_{\mathrm{MO}}$, and $P_{\mathrm{LL}}$, obtained by summing the emission over the regions on the planet's disc as defined in section 3.1. We thus note that these values do not include emission from above the limb, aurora which contributes $\sim 20 \%$ of the total emitted power. The power observed depends strongly on the CML because of the $\sim 10^{\circ}$ tilt of the dipole axis from the spin axis [e.g., Clarke et al., 1980; Prangé et al., 2001]. Following Clarke et al. [1980], the proportion visible from Earth of each auroral region was computed as a function of CML in order to estimate the variation due to CML, and the resulting model flux curves are shown in Figure 2. For each visit, these model flux curves were scaled such that the RMS error between the curves and the measured power values was minimized. The peak value of each scaled flux curve thus represents an auroral activity index for each visit. The deviations of the measured values from the curve were computed and each point was then shifted such that it had this deviation from the curve peak, thus correcting for the variation in viewing geometry caused by the rotation of the planet.

[16] In Figure 2 we also show the entire data set of individual measured power values, where for ease of presentation the values for each visit have been scaled to the single normalized modeled flux curves by dividing them by the above calculated scaling values. This correction method assumes that the auroral power does not vary significantly on the timescale of each visit, and that the auroral emission is roughly homogenous over the entire region. It also does not take into account the geometry of emission from above the limb, since the projection applies to a spheroidal surface, taken to have a radius equal to that of the height of the homopause at auroral latitudes, i.e., $240 \mathrm{~km}$ above the 1 bar level [Vasavada et al., 1999]. The degree to which these assumptions are met varies by auroral region. For example, the morphology of the main oval is generally steady over each visit, whereas the polar auroras are highly variable on the timescale of a HST visit. Similarly, while the morphology of the low-latitude region is generally stable over each HST visit, it is sometimes very patchy and the appearance of a bright region over the limb as the planet rotates may affect the detected flux curve. Figure 2 shows that the correction for CML is most accurate for the main oval, with a root-mean-square error between the scaled power values and the simulated flux curve of 0.06 . The RMS error for the low-latitude region is similar at 0.07 , although the influence of bright patches appearing over the limb can be seen where the measured power increases as the simulated flux curve decreases. The CML correction is less accurate for the polar region, which is highly variable and has an RMS error between the scaled power values and the simulated flux curve of 0.19 . In order to reduce any systematic error introduced by the CML 


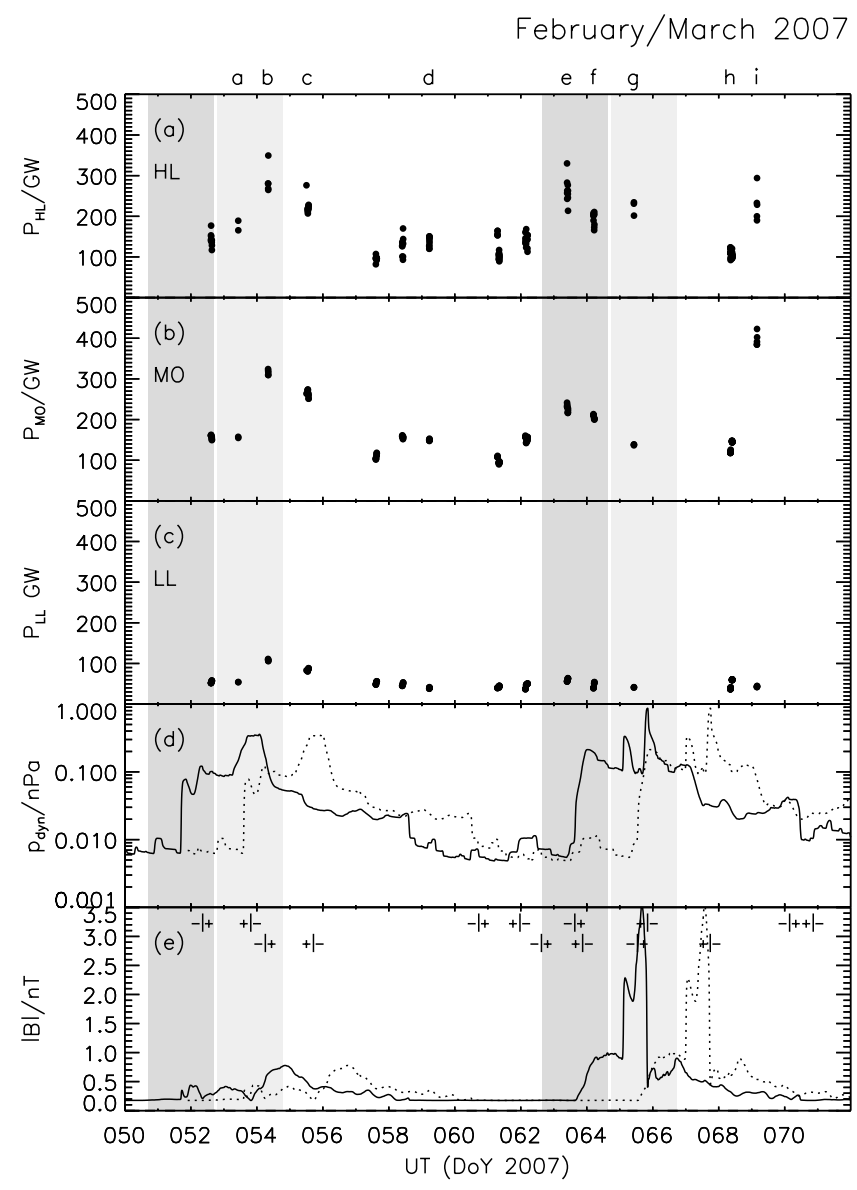

Figure 3. Power emitted from the different auroral regions, along with the modeled solar wind conditions for the first HST campaign in February-March 2007. Specifically, we show (a) the power emitted from the high-latitude region $P_{\mathrm{HL}}$, (b) the power emitted from the main oval region $P_{\mathrm{MO}}$, (c) the power emitted from the low-latitude region $P_{\mathrm{LL}}$, (d) the solar wind dynamic pressure calculated from equation (1), and (e) the interplanetary magnetic field (IMF) magnitude $|B|$. The individual points in Figures $3 a-3 c$ represent the powers obtained for each image. The solid lines in the magnetohydrodynamic (MHD) model (Figures $3 \mathrm{~d}$ and $3 \mathrm{e}$ ) show the original model timings, while the dotted lines show the timings shifted by +2.1 days. The dark gray regions show the estimated arrival time of the forward shocks within 1 standard deviation uncertainty of the MHD model timings, and the light gray regions are similar but for the shifted timings. Also shown in Figure $3 \mathrm{e}$ are the estimated locations of the sector boundaries, along with the sign of $B_{\mathrm{T}}$ either side. The original timing is on top, while the shifted timing is below.

correction, we limit the images used in this study to those with CML between $140^{\circ}$ and $220^{\circ}$, such that the variation due to CML is minimal.

\section{Results}

\subsection{February-March 2007}

[17] In Figure 3 we show the results for the FebruaryMarch 2007 campaign, which coincided with the New
Horizons flyby. Specifically, from top to bottom we show the auroral power emitted from the high-latitude region $P_{\mathrm{HL}}$ (Figure 3a), the auroral power emitted from the main oval region $P_{\mathrm{MO}}$ (Figure $3 \mathrm{~b}$ ), the auroral power emitted from the low-latitude region $P_{\text {LL }}$ (Figure $3 \mathrm{c}$ ), the estimated dynamic pressure $p_{\text {dyn }}$ given by equation (1) (Figure $3 \mathrm{~d}$ ), and the magnitude of the IMF $|B|$ (Figure 3e). All times of HST images have been corrected for the light travel time between Jupiter and Earth. For the solar wind parameters derived from the MHD model the solid line shows the original projection timing, and the dotted line shows the timing including the +2.1 day shift to match the shock on day 53 as observed by New Horizons.

[18] In order to put the variation in power values in context, we first briefly discuss the conditions in the interplanetary medium, estimated by the MHD model. It is important to not concentrate too much on the details of the MHD model result because of the uncertainties in the propagation, although some general points are in order. As discussed by Clarke et al. [2009], the modeled solar wind parameters indicate that two compression regions impinged on Jupiter's magnetosphere during this campaign. These occurred over days 51-58 and 63-70 if one assumes the original model timing is accurate, and 53-60 and 65-72 assuming the shifted times are accurate. These regions are characterized by increased solar wind dynamic pressure of $\sim 10^{-1}$ to $\sim 1 \mathrm{nPa}$, and magnetic field magnitudes of generally a few $\times 10^{-1} \mathrm{nT}$, although peaking at $\sim 3 \mathrm{nT}$ during the second compression. Also shown in Figure $3 \mathrm{e}$ are the times when the model $B_{\mathrm{T}}$ changes sign, with the signs shown on either side of the boundary. The original model timing is shown on top and the shifted times on the bottom. At the sector boundaries the IMF changes from one azimuthal direction to the other. If it goes through the northward direction, the reconnection voltage will be enhanced during this period, such that these intervals may be associated with brightened high-latitude auroras. Of course, the IMF may also go through zero or the southward direction, such that no brightening would be seen in this case.

[19] Clarke et al. [2009] showed that the total emitted UV power exhibited three enhancements, on days 54-55, 6364, and 69-70. The first enhancement was identified as a response to the compression region that impinged on the magnetosphere on day 53. The trigger of the second enhancement is somewhat unclear, because of the uncertainty in the MHD model timing. Assuming the original timing is accurate, the enhancement corresponds to the forward shock of the second compression region, estimated to impinge on Jupiter on day 63. However, maintaining the +2.1 day shift places the enhancement in the middle of the previous rarefaction region. The third enhancement was identified as a dawn storm event, and occurred toward the middle to end of the second compression region, depending on the model timing.

[20] Figure 3 shows that these three enhancements in the total emitted power are evident to a greater or lesser extent in all auroral regions. Considering first the main oval, the emitted power $P_{\mathrm{MO}}$ shown in Figure $3 \mathrm{~b}$ has a mean of $\sim 170 \mathrm{GW}$ and standard deviation of $\sim 70 \mathrm{GW}$, i.e., $\sim 40 \%$ of the mean. It exhibits "baseline" values of $\sim 100-$ $200 \mathrm{GW}$, with the power increasing to $\sim 200-400 \mathrm{GW}$ 
(a) $05311: 14: 46 \quad C M L=142.4$

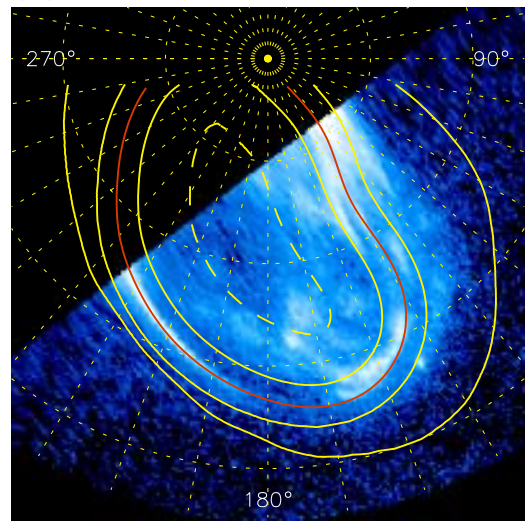

(d) $05906: 16: 20 \quad C M L=144.7$

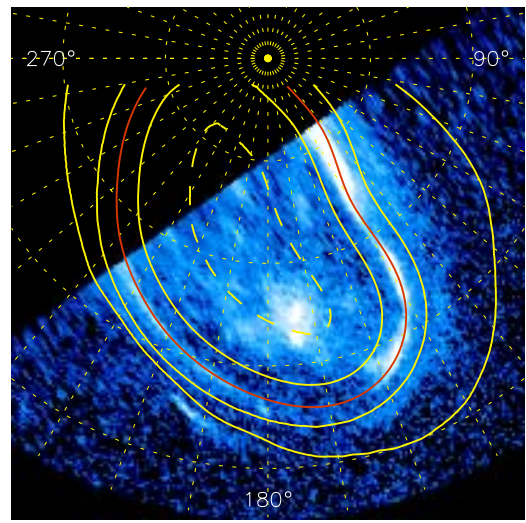

(g) $06511: 00: 46 \quad \mathrm{CML}=140.1$

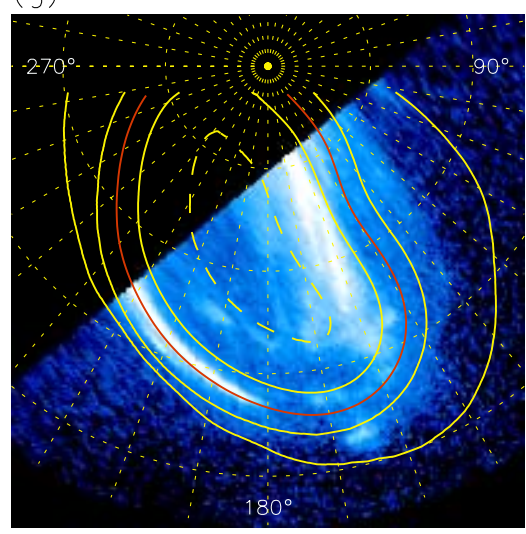

(b) $05408: 55: 53 \quad \mathrm{CML}=209.0$

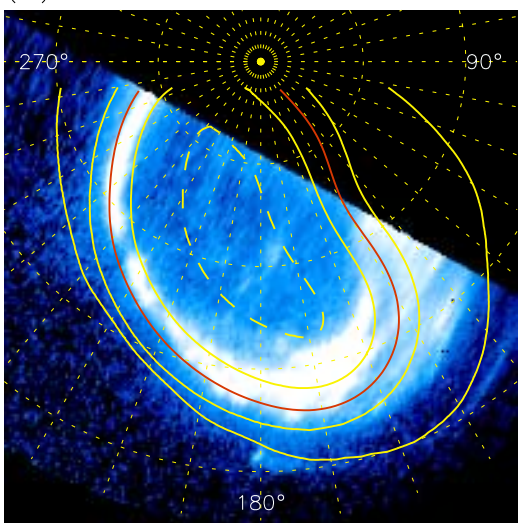

(e) $063 \quad 10: 23: 41 \quad C M L=176.6$

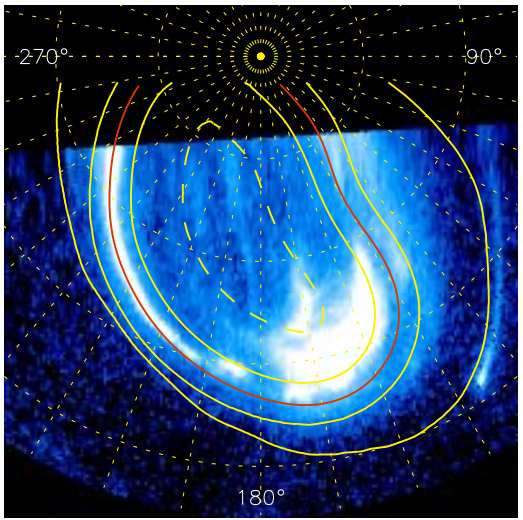

(h) $068 \quad 09: 19: 29 \quad \mathrm{CML}=170.1$

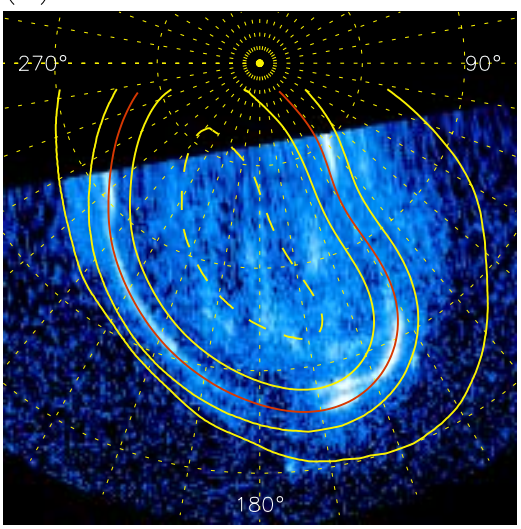

(c) $055 \quad 13: 51: 47 \quad \mathrm{CML}=178.4$

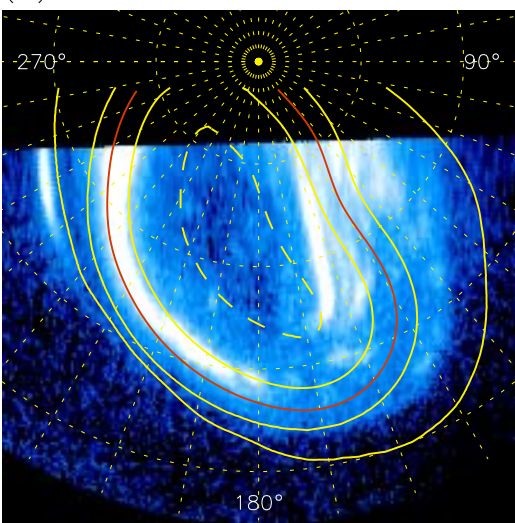

(f) 064 06:13:19 $\quad \mathrm{CML}=175.8$
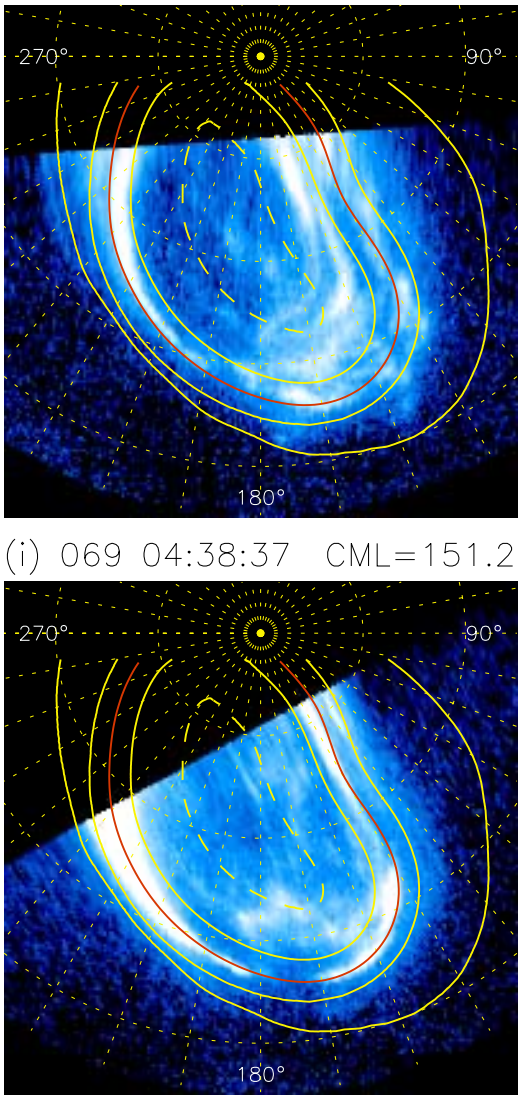

Figure 4. Selected images, which correspond to the visits labeled at the top of Figure 3. The format of each is as for Figure 1.

during the three enhancements discussed above. A selection of images corresponding to the visits labeled at the top of Figure 3 are shown in Figure 4. During "quiet" times the main oval is generally located along the reference oval, although between days 53 and 54 the main oval increased in brightness and moved poleward $\sim 1^{\circ}$ in the narrow, welldefined sector greater than SIII longitude $\sim 180^{\circ}$, and expanded to merge with the high-latitude auroras over smaller longitudes, as shown in Figures $4 \mathrm{a}$ and $4 \mathrm{~b}$. This brightness increase is particularly evident over longitudes greater than $\sim 165^{\circ}$, with smaller longitudes exhibiting only minor, if any, brightening within the defined main oval strip. As indicated by the elevated $P_{\text {MO }}$ values in Figure 3 and the image shown in Figure 4c, the main oval remained bright and expanded over longitudes greater than $\sim 180^{\circ}$ until at least day 55. The remainder of the main oval region is very faint, to the point of there being no well-defined main oval emission in this region. The main oval emitted power then returned to its quiet state, as shown by the example image in Figure 4d, with emitted power values of $\sim 100-200 \mathrm{GW}$, and remained this way until day 63 when it again brightened over longitudes greater than $\sim 165^{\circ}$, as shown in Figure $4 \mathrm{e}$. The oval remained bright and somewhat disturbed over day 64 , as shown in Figure 4f, and by day 65, shown in Figure 4g, exhibited similar morphology to the day 55 image shown in Figure 4c. By day 68 the oval had 
February/March 2007
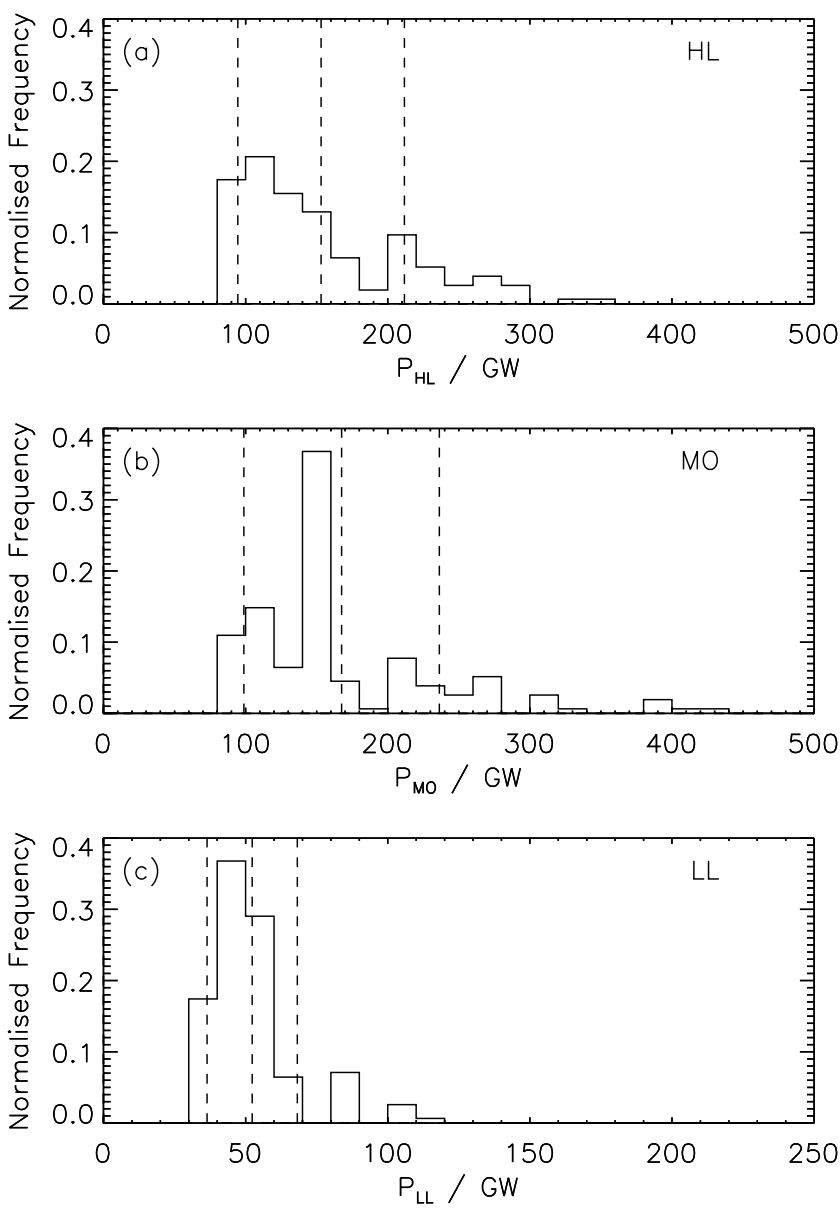

Figure 5. Distributions of the powers shown in Figure 3. The middle vertical dashed line shows the mean of the distribution, while the outer vertical lines show \pm 1 standard deviation. Note the expanded horizontal scale in Figure 5c.

returned to lower brightness, as shown in Figure 4h, and the following day brightened in a dawn storm event, in which the main oval brightened along the majority of its length, especially on the dawnside where it brightened and expanded to fill the whole $4^{\circ}$ main oval strip. The distribution function of $P_{\mathrm{MO}}$ is shown in Figure $5 \mathrm{~b}$, along with the above mean and standard deviation range, shown by the vertical dashed lines. The most frequent power values lie in the range $140-160 \mathrm{GW}$, with a frequency of $\sim 35 \%$. The remainder of the distribution is skewed positive, and there is a suggestion of bimodality corresponding to the enhanced states with power values $>\sim 200 \mathrm{GW}$ versus the "baseline" power values of $\sim 100-200 \mathrm{GW}$. The $\sim 400 \mathrm{GW}$ highpower tail corresponds to the day 69 dawn storm.

[21] The power emitted from the low-latitude auroras $P_{\mathrm{LL}}$, shown in Figure 3c, has a mean of $\sim 50 \mathrm{GW}$ and a standard deviation of $\sim 15 \mathrm{GW}$, i.e., $\sim 15 \%$ of the mean. It exhibits "baseline" values of $\sim 50 \mathrm{GW}$, and increased to $\sim 100 \mathrm{GW}$ along with the main auroral oval during the first enhancement event. The majority of this increased power is due to brightened emission in the "kink" region near $\sim 140^{\circ}$ longitude, evident in, e.g., Figure 4c. There is a suggestion that this region brightened slightly during the second main oval brightening, in Figure 4e, although the power increase is much less in this case, to $\sim 75 \mathrm{GW}$.

[22] The low-latitude auroras did not increase in brightness during the day 69 dawn storm event. The distribution of $P_{\mathrm{LL}}$ shown in Figure $5 \mathrm{c}$ is also skewed positive, although to a lesser extent than the main oval since this region did not greatly increase in brightness during the second and third main oval brightening events.

[23] The power emitted from the high-latitude region $P_{\mathrm{HL}}$, shown in Figure 3a, exhibits a similar long-term variation to the main oval, although there is significant variation superposed thereon. The changeable nature of the polar auroras is evident in the vertical spread of power values obtained during each visit. The mean value of $P_{\mathrm{HL}}$ is $\sim 155 \mathrm{GW}$, and the standard deviation is $\sim 60 \mathrm{GW}$, i.e., $\sim 40 \%$ of the mean, such that although the power variation in each visit is larger for the high-latitude auroras, the overall relative variation is similar to that for the main oval. The "baseline" power values are $\sim 100-200 \mathrm{GW}$, which is emitted from the variable, patchy emission continually present in the polar region, such as in the image in Figure $4 \mathrm{~h}$. The variation in power is due to transient blobs of emission, such as those evident in Figures 4a, 4d, and 4i, which appear and disappear over a few minutes. They occurred throughout the campaign, both when the main oval was dim, (e.g., Figure 4d) and bright (e.g., Figure 4e). The long-term variation in the polar auroras is due to bright arcs, which occur just poleward of the main oval in the postnoon sector, and are stable over at least $3 \mathrm{~h}$. On days 54 and 63 the region contiguous to the main auroral oval between $\sim 145-175^{\circ}$ brightened along with main oval, as shown in Figures $4 \mathrm{~b}$ and $4 \mathrm{e}$. In each enhancement event, during the 2 days following the initial power increase bright arcs were evident on the duskside, e.g., in Figures 4c, 4f, and $4 \mathrm{~g}$. Occasionally multiple arcs were observed, as in Figure $4 \mathrm{f}$. In order to show the local time dependence of the poleward arcs, we show in Figure 6 the example that occurred on day 55. On this day, five visits were made such that images with a wide range of CMLs were obtained, and in Figure 6 we show samples from the whole CML range of $\sim 140^{\circ}$ to $\sim 236^{\circ}$, with the CML oriented toward the bottom in each case. The bright arc poleward of the main oval can be seen in each panel, and in all cases its location is on the duskside, despite the planet rotating $\sim 100^{\circ}$ throughout the sequence. The high-latitude auroras also brightened during the dawn storm event on day 69, although there was considerable variation in emitted power during this visit, from $\sim 180-380 \mathrm{GW}$. The distribution of $P_{\mathrm{HL}}$ shown in Figure 5a is, like for $P_{\mathrm{MO}}$ and $P_{\mathrm{LL}}$ skewed positive, and is also bimodal about $\sim 200 \mathrm{GW}$, with the two populations corresponding to the baseline and enhanced states.

[24] As discussed in section 3.1, the high-latitude region was further divided into the polar inner and polar outer regions, and the respective emitted powers $P_{\mathrm{PI}}$ and $P_{\mathrm{PO}}$ computed, in order to characterize the variation of the swirl region auroras without contamination from the bright poleward arcs. The results are shown in Figure 7, in which we show $P_{\mathrm{PI}}($ Figure $7 \mathrm{a})$ and $P_{\mathrm{PO}}$ (Figure $7 \mathrm{~b}$ ), along with $P_{\mathrm{HL}}$ (Figure 7c) and $P_{\mathrm{MO}}$ (Figure 7d) for comparison. The mean and standard deviation of $P_{\mathrm{PI}}$ are $\sim 40 \mathrm{GW}$ and $\sim 9 \mathrm{GW}$, 
(a) 055 12:48:08 $\quad \mathrm{CML}=139.9$

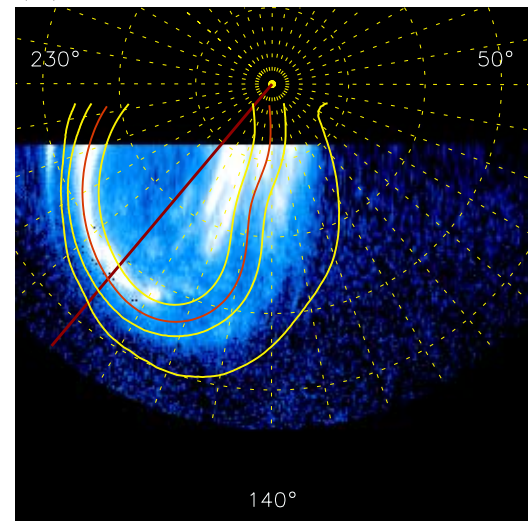

(d) 055 13:54:25 $\quad \mathrm{CML}=179.9$

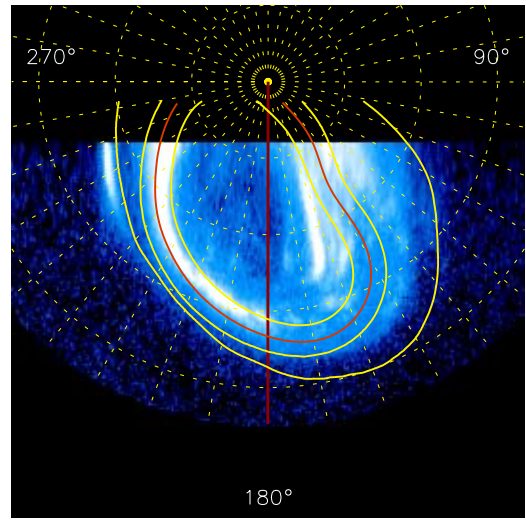

(g) 055 14:25:03 CML=198.5

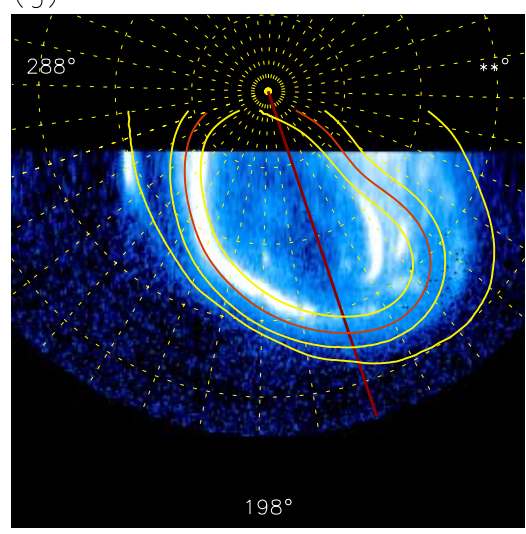

(b) $055 \quad 12: 50: 28 \quad \mathrm{CML}=141.3$

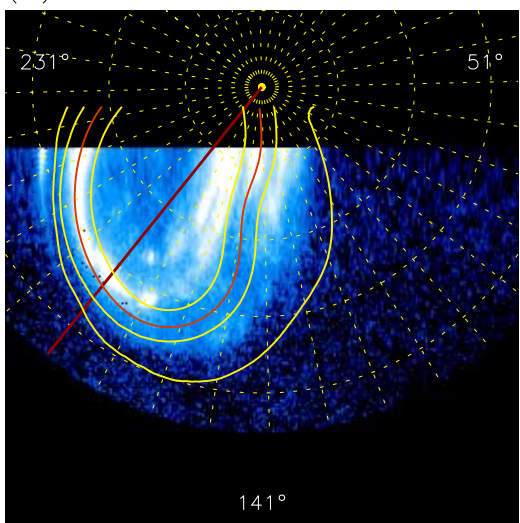

(e) 055 14:03:45 CML=185.6

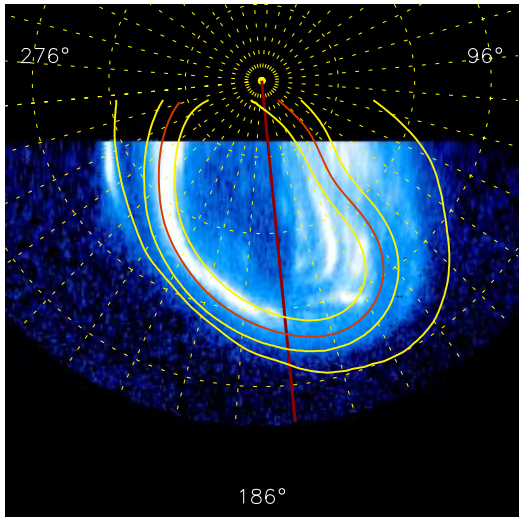

(h) 055 15:23:04 CML=233.5

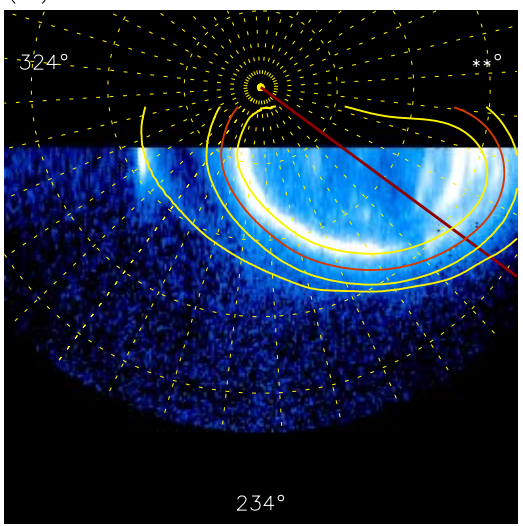

(c) $055 \quad 13: 42: 27 \quad C M L=172.7$

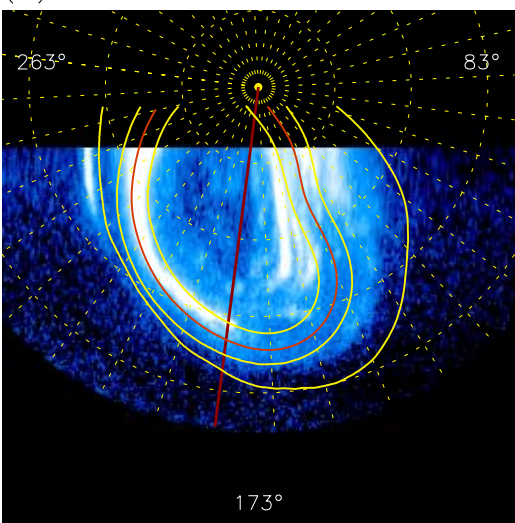

(f) 055 14:20:23 CML=195.6

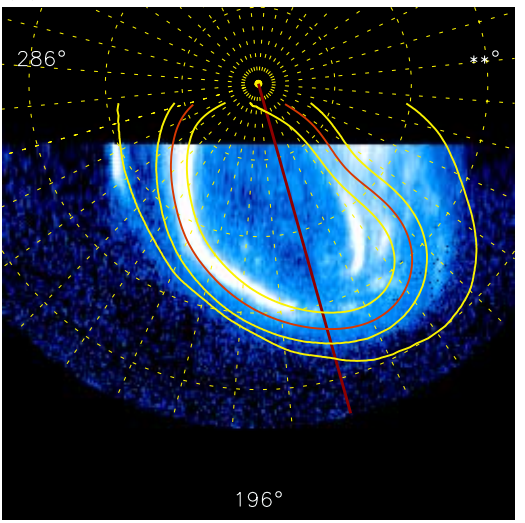

(i) $055 \quad 15: 27: 44 \quad \mathrm{CML}=236.4$

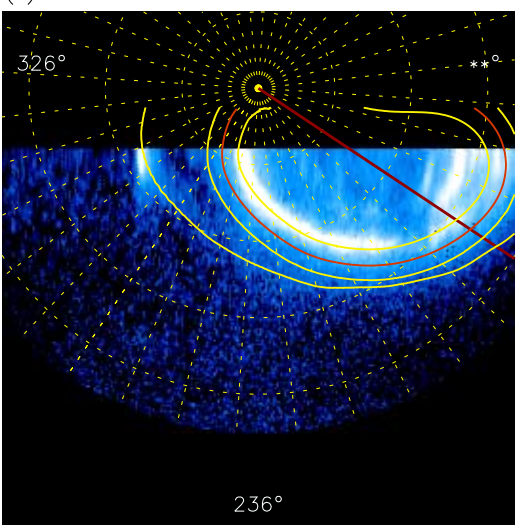

Figure 6. Representative images obtained on day 55 covering a large CML range of $\sim 100^{\circ}$. The format is as for Figure 1, except that in each case the CML is oriented toward the bottom, and for reference the $180^{\circ}$ SIII meridian is shown in red.

respectively, while for $P_{\mathrm{PO}}$ they values are $\sim 110 \mathrm{GW}$ and $\sim 55 \mathrm{GW}$. It is evident that, although both polar regions are highly variable, the outer region varies essentially with the main auroral oval, while the inner region shows no such correlation except for possibly during the day 69 dawn storm, in which the polar inner auroras (free from any possible contamination from expanded main oval emission) increased in emitted power to $\sim 50-70 \mathrm{GW}$.

[25] In order to determine formally whether the different auroral regions vary in cohesion we show in Figure 8 the power components $P_{\mathrm{HL}}$ (Figure $8 \mathrm{a}$ ), $P_{\mathrm{LL}}$ (Figure $8 \mathrm{~b}$ ), $P_{\mathrm{PI}}$ (Figure $8 \mathrm{c}$ ), and $P_{\mathrm{PO}}$ (Figure $8 \mathrm{~d}$ ) plotted versus the main oval power $P_{\mathrm{MO}}$, and in each case show the linear correlation coefficient $r$. The correlation coefficient for the highlatitude auroras of 0.80 indicates reasonable correlation, and the lower value of 0.56 for the low-latitude emission correlation coefficient arises since this emission did not increase greatly during the second main oval brightening event. The subregions of the high-latitude auroras indicate that, as discussed above, the polar outer auroras, with a correlation coefficient of 0.79 , are responsible for the correlation with the main oval, while the polar inner auroras, with a correlation coefficient of 0.38 , vary independently. 


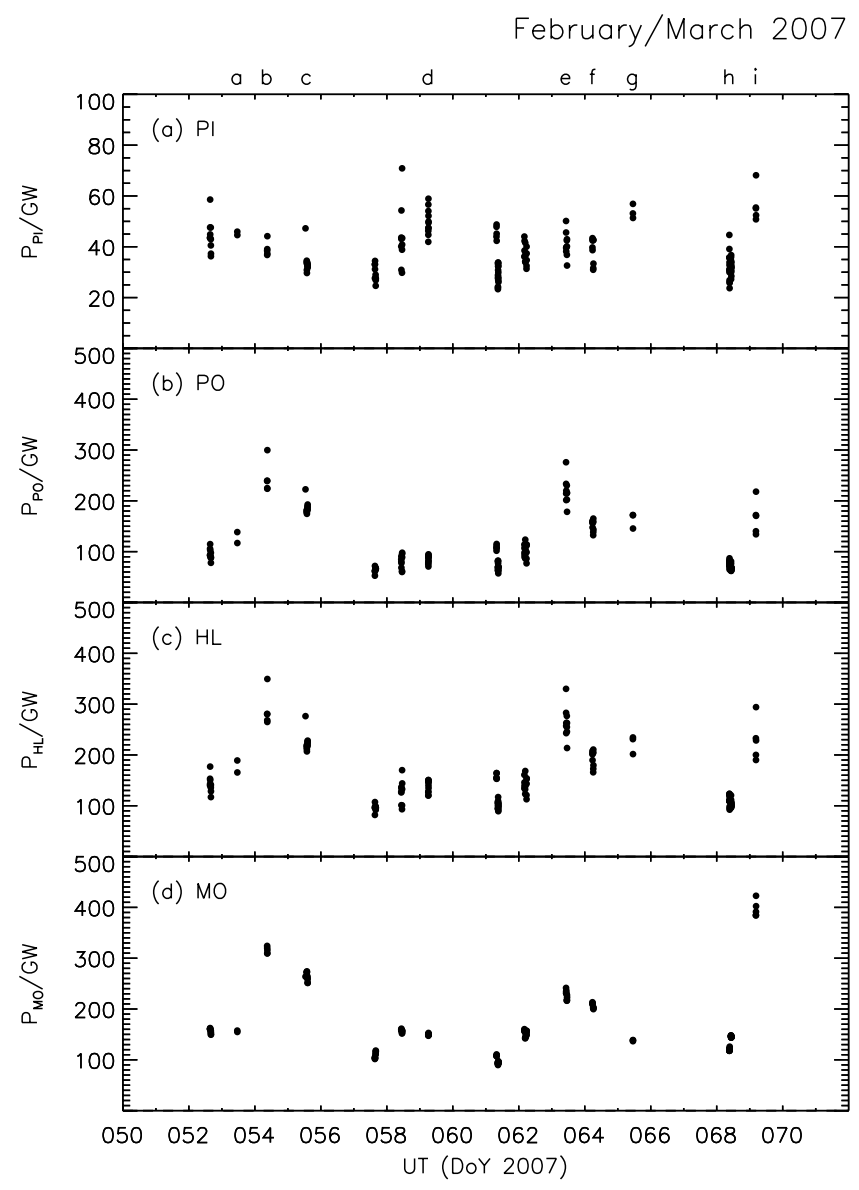

Figure 7. (a) Power emitted from the polar inner region $P_{\mathrm{PI}}$ and (b) power emitted from the outer polar region $P_{\mathrm{PO}}$, along with (c) power emitted from the whole high-latitude region $P_{\mathrm{HL}}$ and (d) power emitted from the main oval $P_{\mathrm{PO}}$ for comparison. Note the expanded vertical scale in Figure $7 \mathrm{a}$.

\subsection{May-June 2007}

[26] Turning now to the second campaign from May-June 2007, when the planet was near opposition, we show in Figure 9 the results in a similar format to Figure 3, although note that in this case only one model timing is shown since there are no in situ measurements with which to compare the model. Again, sample images corresponding to the labeled visits are shown in Figure 10. As discussed by Clarke et al. [2009], the propagated interplanetary medium properties indicate that four forward shocks impinged on the planet during this campaign, on days 134, 143, 153, and 158. The pressure and magnetic field estimates indicate that the first occurred during a compression region which lasted until day 136, the second occurred at the onset of a second compression region which lasted until day 151, the third then occurred at the start of a third compression after a short $\sim 2$ day rarefaction region, and the fourth occurred within this last compression, which lasted until day 161. The magnitude of the estimated IMF peaked at $\sim 3 \mathrm{nT}$ during the first and third compression regions, but only reached $\sim 0.5 \mathrm{nT}$ during the second compression region.
[27] Clarke et al. [2009] showed that during the second campaign the total emitted UV power exhibited more complex behavior than during the first. Enhancements were observed that may be associated with the first, third and fourth forward shocks, and there occurred a dawn storm $\sim 2$ days before the second. There was a HST data gap following the second shock, and although no enhancement was seen during the predicted arrival time of the shock the power values after the gap were elevated, suggesting the shock might have impinged on the magnetosphere during the gap. Clarke et al. [2009] showed, however, that there were enhancements, e.g., on day 138, that occurred well within rarefaction regions, and conversely some of the dimmest auroras occurred during compression regions, e.g., on day 133 .

[28] Considering first the power emitted by the main auroral oval $P_{\mathrm{MO}}$, shown in Figure $9 \mathrm{~b}$, the behavior is apparently more complex than for the first campaign. The mean power is $\sim 130 \mathrm{GW}$, significantly lower than for February-March, and since there are no enhancements to the $\sim 400 \mathrm{GW}$ level as observed in February, the standard deviation is slightly lower at $\sim 50 \mathrm{GW}$. Interestingly, as shown in Figure 10, throughout the second campaign, where the main oval is well defined it is generally $\sim 1^{\circ}$ equatorward of the reference main oval, possibly indicating a change in the current sheet configuration between the two campaigns, such as that discussed by Grodent et al. [2008b]. The main oval initially exhibits a moderate emitted power of $\sim 150 \mathrm{GW}$, and as can be seen in Figure 10a is again observed to be narrow and well defined over longitudes greater than $\sim 180^{\circ}$, and diffuse over smaller longitudes. The emitted power then decreased over the next few days to very low values of $\sim 50 \mathrm{GW}$, corresponding to a very faint oval, at least over longitudes greater than $\sim 160^{\circ}$ as shown in Figure 10b. The emitted power then increased to $\sim 200 \mathrm{GW}$ on day 134 , possibly associated with the significant pressure enhancement that occurred around this time. As shown in Figure 10c this increase in power is associated with bright patches along the oval, and an expansion from the low-latitude boundary of the defined main oval strip toward the reference oval. The emitted power then decreased again over the next few days to $\sim 100 \mathrm{GW}$, until a small increase again to $\sim 180 \mathrm{GW}$. After a few day data gap, the main oval exhibits a dawn storm event on day 142, as shown in Figure 10d in which the main oval brightened in the dawn sector, although the rest of the oval was faint at this time such that the emitted power is $\sim 200 \mathrm{GW}$, rather than the $\sim 300-400 \mathrm{GW}$ of the day 69 dawn storm. The dawn storm was gone by the following day, and there followed another few day data gap, during which the second compression region impinged on Jupiter, according to the MHD model. During this compression region the main oval underwent another dawn storm event on day 149, as shown in Figure 10f, in which the main oval exhibited two bright arcs in the dawn which doubled the emitted power from $\sim 100 \mathrm{GW}$ to $\sim 200 \mathrm{GW}$. During the final compression region the main oval exhibited bright but patchy emission, such as in Figure $10 \mathrm{~g}$, in which a well defined oval does not exist, but where much of the main oval region is dark except for some bright patches which merge with similar forms in the low-latitude region. There also occurred a second forward shock during this compres- 

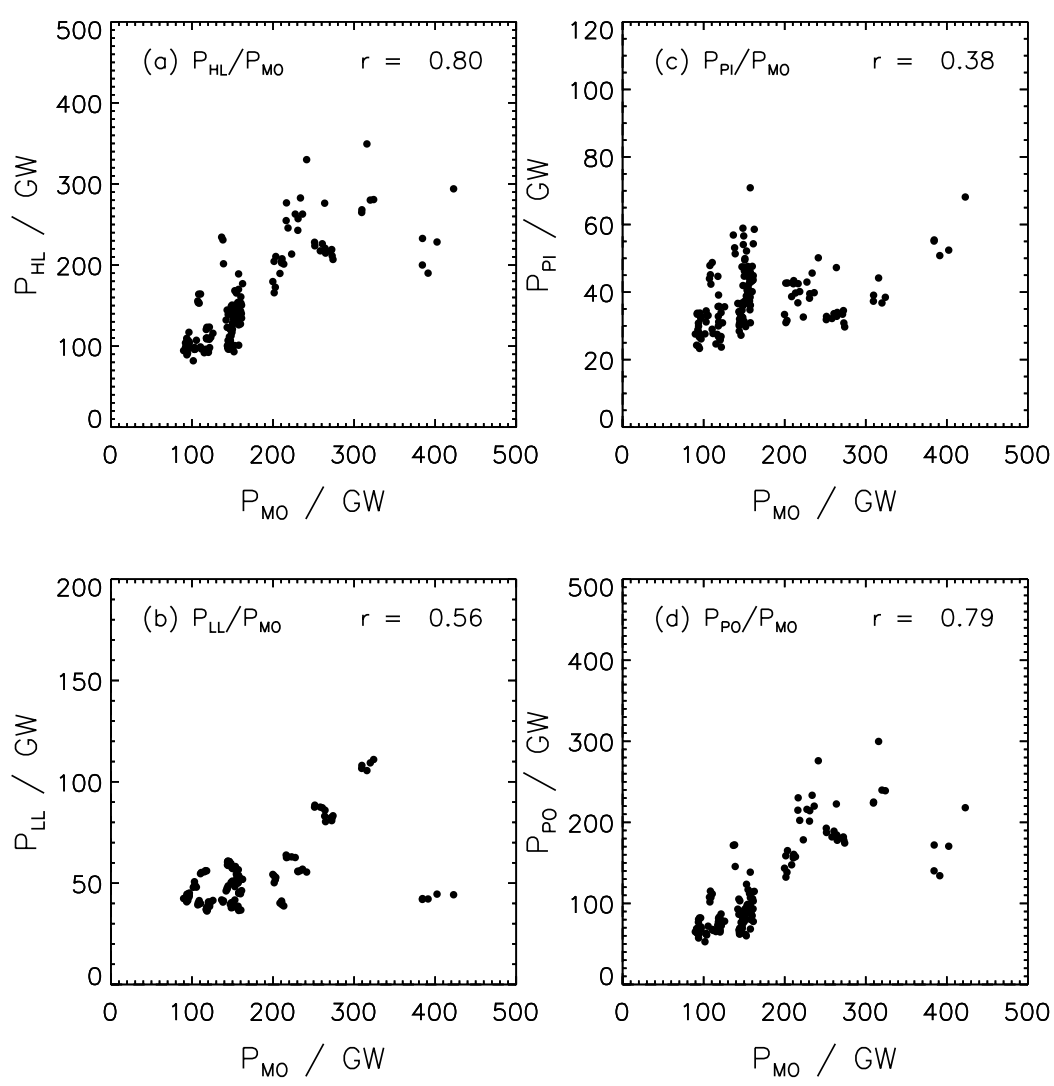

Figure 8. (a) $P_{\mathrm{HL}}$, (b) $P_{\mathrm{LL}}$, (c) $P_{\mathrm{PI}}$, and (d) $P_{\mathrm{PO}}$ versus $P_{\mathrm{MO}}$, along with the values of the correlation coefficient. Note the panels have different vertical scales.

sion region, in response to which, as shown in Figure 10h, the main oval exhibited morphology similar to that observed in response to forward shocks in the first campaign, e.g., in Figures $4 \mathrm{~b}, 4 \mathrm{c}, 4 \mathrm{e}$, and $4 \mathrm{~g}$, in which the oval is bright over longitudes greater than $\sim 180^{\circ}$, and at lower longitudes is dimmer along the reference oval, but instead there is emission which appears to merge with bright poleward emission in the afternoon sector. Finally, during the rarefaction region that followed, as shown in Figure 10i the main oval reverted to an orderly state in which the emission was narrow and well defined along its entire length, and the emitted power was $\sim 100 \mathrm{GW}$. The distribution of $P_{\mathrm{MO}}$ for the second campaign shown in Figure $11 \mathrm{~b}$ is not skewed positive as before, but is instead roughly flat between $\sim 40-$ $220 \mathrm{GW}$.

[29] The low-latitude emission was generally much brighter during the second campaign than the first, with a mean emitted power $P_{\mathrm{LL}}$ of $\sim 85 \mathrm{GW}$. It is also more variable, with a standard deviation of $35 \mathrm{GW}$, i.e., $\sim 40 \%$ of the mean. While for the first campaign there was generally no significant emission in the defined low-latitude region, except for in the kink region as shown in Figures 4a and $4 \mathrm{~b}$, in the second campaign significant bright emission was observed at all longitudes in the low-latitude region. This took the form of diffuse emission, as in Figure 10a, bright patches, as in Figures 10c, 10f, and 10g, or secondary ovals, as shown in Figures $10 \mathrm{~b}$ and 10e. These bright features elevated the emitted power to values $\sim 150 \mathrm{GW}$, and the variation was generally in step with the main oval emitted power. The major exception to this is the event on day 154 in which the low-latitude emitted power reached $\sim 200 \mathrm{GW}$, the brightest of either campaign, caused by the multiple bright patches shown in Figure $10 \mathrm{~g}$, and which was not accompanied by a similar large increase in $P_{\mathrm{MO}}$. The distribution of $P_{\mathrm{LL}}$ shown in Figure 11c is much broader than for the first campaign, such that, although the power values are mostly clustered between $\sim 40-100 \mathrm{GW}$, the higher-power tail is much longer, corresponding to the brightenings such as on day 154 .

[30] While the low-latitude emission was generally brighter during the second campaign, the opposite is true for the high-latitude auroras, which have a mean emitted power value of $\sim 80 \mathrm{GW}$, almost half that of the first, and standard deviation $\sim 35 \mathrm{GW}$, i.e., a relative variation of $\sim 40 \%$, similar to the first campaign. Unlike the first campaign, the long-term variation of $P_{\mathrm{HL}}$ does not exhibit correlation with $P_{\mathrm{MO}}$. Overall, however, similar morphological features are present in the second campaign as for the first, for example the multiple poleward arcs on the afternoon-duskside as in Figures 10a and 10h, the bright active region blobs as shown in Figures $10 \mathrm{~b}$ and $10 \mathrm{~h}$, and the diffuse emission present to different degrees in all images. As with the first campaign, these may be features that occur over $\sim 2$ days following a forward shock, such as 


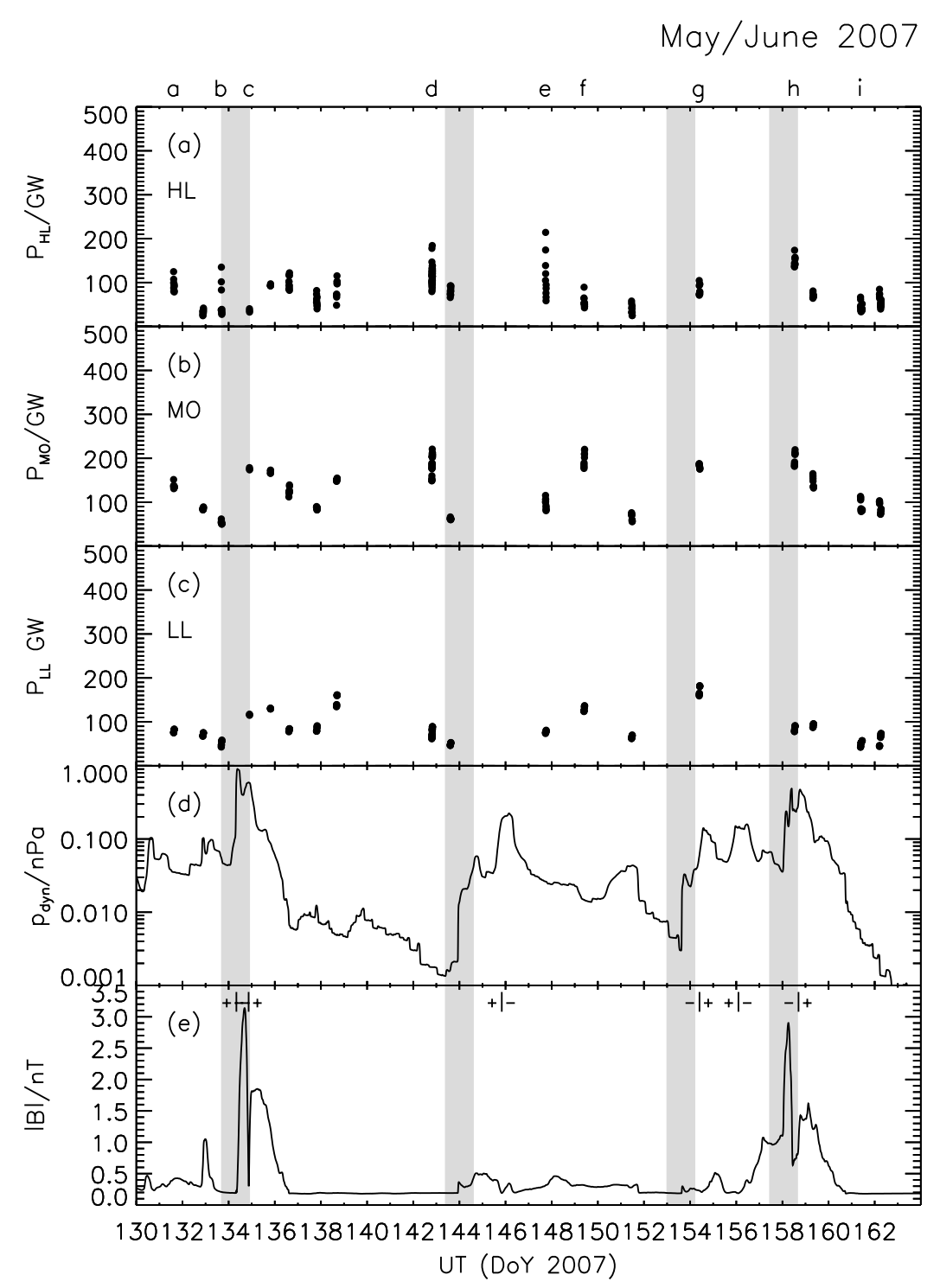

Figure 9. As for Figure 3, but for May-June 2007. Note that here there is no shifted MHD timing. The vertical axis scales are identical to Figure 3 for ease of comparison.

for the examples in Figures 10a and 10h. We should also note, however, that, propagation timing uncertainties and the data gap over days 144-147 notwithstanding, there occurred two forward shocks which did not exhibit cohesive, long-lived poleward arcs, so this association is not certain. The distribution of $P_{\mathrm{HL}}$ shown in Figure 11a highlights the lower mean power of $P_{\mathrm{LL}}$ with respect to the first campaign, and also does not exhibit the bimodality of the first campaign. Instead the power values cluster between $\sim 40-100 \mathrm{GW}$, falling off thereafter to $\sim 200 \mathrm{GW}$. $\sim 40-$ $220 \mathrm{GW}$.

[31] The power emitted from the subregions of the highlatitude auroras, $P_{\mathrm{PI}}$ and $P_{\mathrm{PO}}$ is shown in Figure 12. Both regions have decreased emitted power compared to the first, with mean values of $\sim 25 \mathrm{GW}$ and $\sim 55 \mathrm{GW}$ for $P_{\mathrm{PI}}$ and $P_{\mathrm{PO}}$, respectively. The standard deviation values of $\sim 9 \mathrm{GW}$ and $\sim 30 \mathrm{GW}$ for $P_{\mathrm{PI}}$ and $P_{\mathrm{PO}}$, respectively, represent $\sim 40 \%$ and $\sim 55 \%$ of the mean, i.e., larger relative variation than for the first campaign in both cases. The dawn storm on day 142, shown in Figure 10d, is particularly prominent in all traces, again showing that the power emitted by all individual auroral components increases during these events. Also prominent are the bright, transient blobs that occurred in the inner polar region on day 133, shown in Figure 10b, and the outer polar region on day 147, shown in Figure 10e.

[32] Finally, plots of $P_{\mathrm{HL}}, P_{\mathrm{LL}}, P_{\mathrm{PI}}$, and $P_{\mathrm{PO}}$ versus $P_{\mathrm{MO}}$ are shown in Figure 13, along with the correlation coefficients. The value of the latter of 0.57 for $P_{\mathrm{HL}}$ indicates weak correlation at best between the high-latitude auroras and the main oval, significantly different from the first campaign which showed relatively good correlation between these two components. Despite the differences in the morphology and brightness of the low-latitude emission, between the first and second campaign, the correlation with the main oval is remarkably similar, at 0.57 for the second campaign versus 0.56 for the first. The polar inner region, with a correlation coefficient of 0.38 also exhibits similar correlation in both campaigns, but the polar outer auroras have a much lower value of 0.55 , indicating this region does 
(a) $131 \quad 15: 53: 11 \quad \mathrm{CML}=177.3$

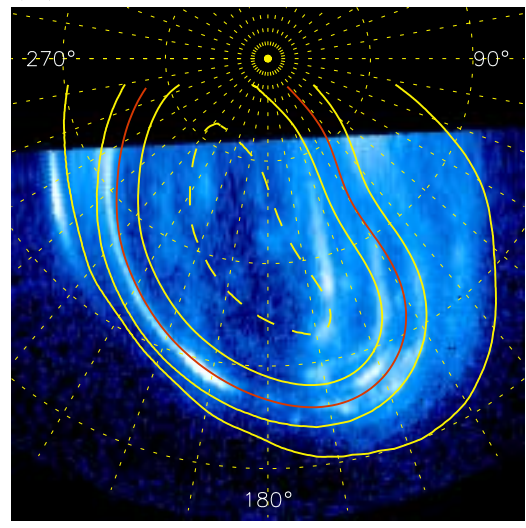

(d) $142 \quad 20: 26: 43 \quad C M L=199.8$

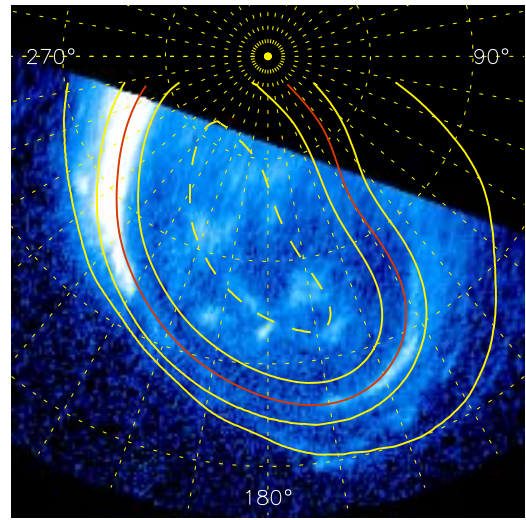

(g) $154 \quad 10: 01: 05 \quad \mathrm{CML}=189.9$

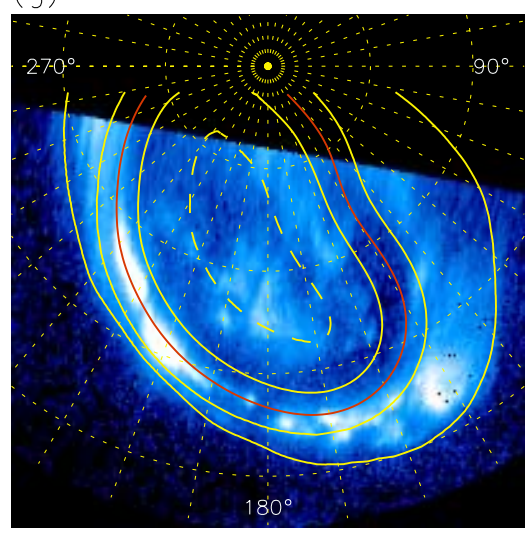

(b) $133 \quad 17: 02: 00 \quad \mathrm{CML}=160.2$

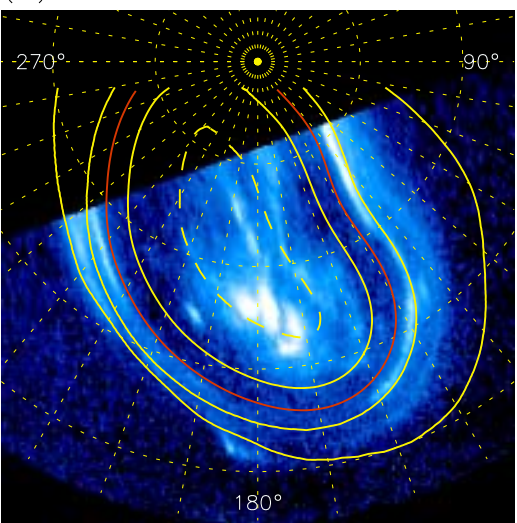

(e) 147 18:18:10 $\mathrm{CML}=155.8$
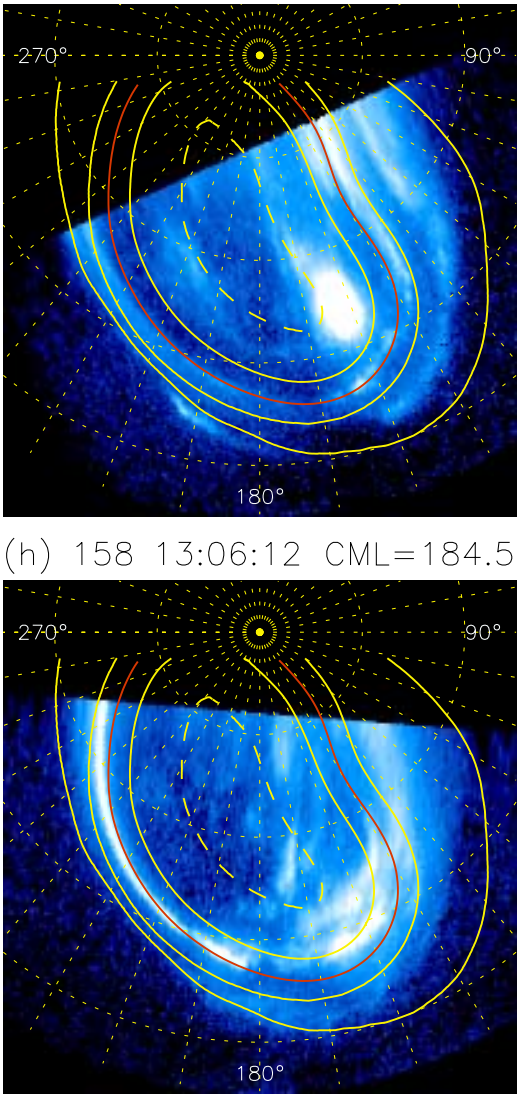

(c) 134 22:21:21 $\quad \mathrm{CML}=144.0$
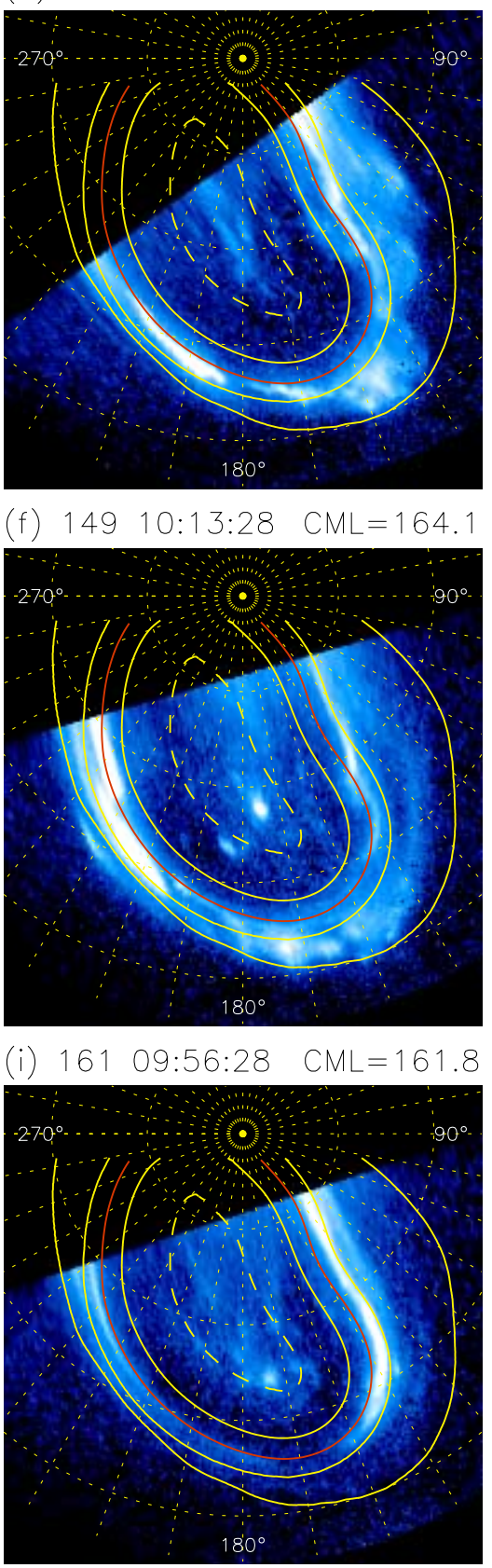

Figure 10. As for Figure 4, but for May-June 2007.

not have the same direct relation to the main oval as observed for the first campaign.

\section{Results Using a Dynamic Definition of the Main Oval}

[33] We finally present in Figure 14 the results using the dynamic definition of the main oval. Figures $14 \mathrm{a}$ and $14 \mathrm{~d}$ show that the deviation from the reference oval is generally a degree or less but, as discussed in section 4, during intervals associated with solar wind compression regions it is located $\sim 0.5-1^{\circ}$ poleward of it location during rarefaction regions. Also, as discussed in section 4.2, during the second campaign its location is generally $\sim 0.5-1^{\circ}$ equatorward of the reference main oval, although approaching $\sim 2^{\circ}$ on day 151 . The powers emitted from the main oval and high-latitude regions are shown in Figures $14 \mathrm{c}$ and $14 \mathrm{f}$ and Figures $14 \mathrm{~b}$ and 14e, respectively, and the RMS of the difference between the results using the fixed oval and those using the dynamic oval is $\sim 10 \%$, comparable to the uncertainty in the CML correction algorithm. We conclude that the results obtained using the fixed oval definition accurately reflect the behavior of the oval.

\section{Summary of Observations}

[34] In this paper the extensive HST data set obtained over two month-long campaigns in 2007 was used to 
May/June 2007
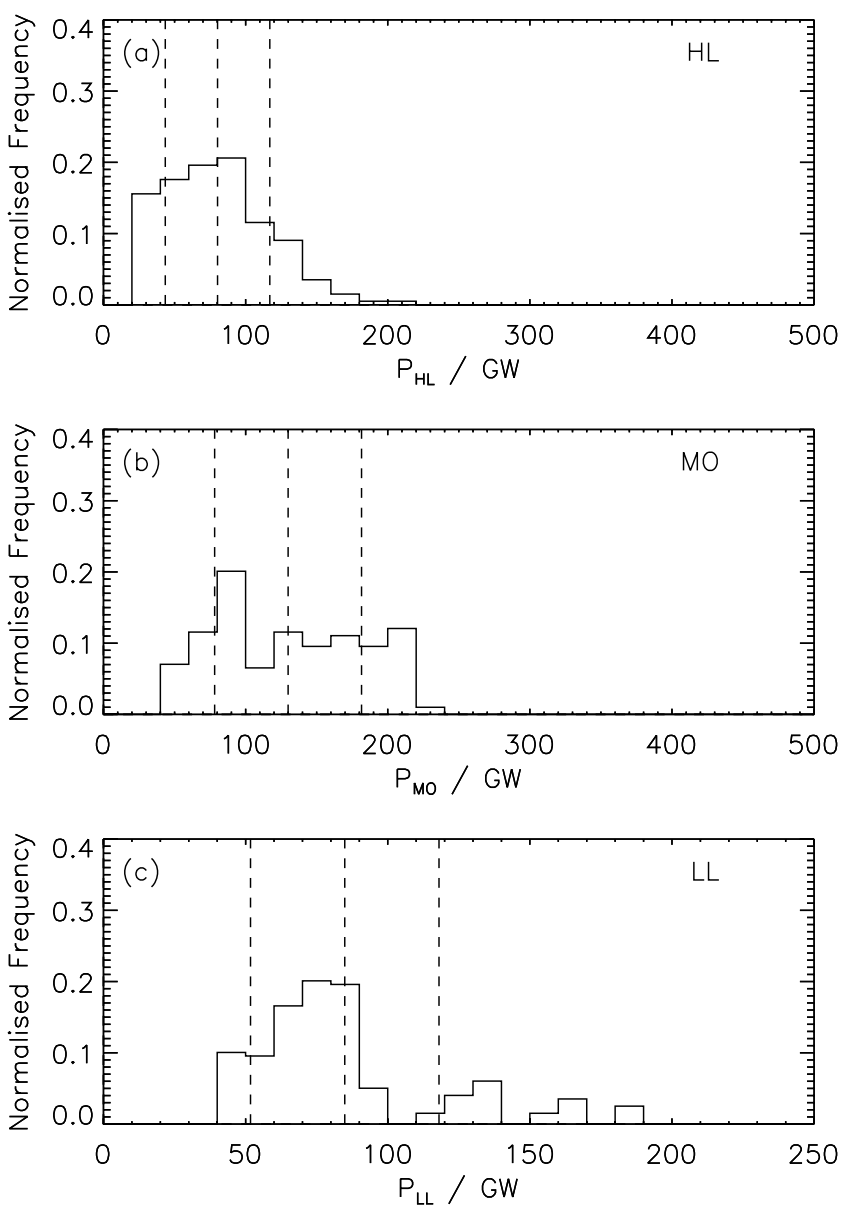

Figure 11. As for Figure 5, but for May-June 2007. Note again the expanded horizontal scale in Figure 11c.

determine the long-term variability of the different components of Jupiter's auroras. The UV auroral power emitted from three regions on the planet's disc, i.e., the main oval, low-latitude, and high-latitude auroras, was extracted. The latter was further divided into the polar inner and polar outer regions. The temporal variation of these parameters was discussed with reference to the auroral morphology and estimated solar wind conditions projected to Jupiter from data obtained at Earth orbit. A summary of the observations is as follows:

[35] 1. The morphology and emitted powers varied greatly between the February-March and May-June campaigns.

[36] 2. In the first campaign the emitted power originated mainly from the main oval and the high-latitude regions, which roughly correlated, and both of which displayed two distinct states, quiet and enhanced.

[37] 3. In the second campaign the high-latitude and main oval auroras were generally dimmer overall and less variable, while the low-latitude region was populated with bright, patchy emission.

[38] 4. With the exception of the main oval and polar outer auroras during the first campaign, discussed below, it was shown that the different auroral regions exhibited weak or no correlation, indicating different source mechanisms.
[39] 5. Transient blobs of polar emission occurred throughout the campaigns, i.e., during intervals of both rarefied and compressed solar wind, and the very high latitude auroras exhibited behavior independent to the other components.

[40] 6. A specific type of enhancement event is associated with compression regions; that is, over longitudes greater than $\sim 180^{\circ}$ the main oval is bright and located $\sim 1^{\circ}$ poleward of its previous location, while over smaller longitudes the main oval is not bright or well defined. Instead there was bright emission originating from the contiguous poleward region in the postnoon and dusk sectors where bright, sometimes multiple arcs formed. This state is repeatedly maintained for up to $\sim 2$ days.

[41] 7. Dawn storm events occurred during solar wind compression and rarefaction regions, and it was shown that the high-latitude auroras brightened as well as the main auroral oval.

\section{Discussion}

[42] The first campaign was characterized by three clear enhancement events during which the power emitted from the main oval and high-latitude regions increased. The first two of these events exhibited similar auroral morphology, described in point 6 above, to that observed once during the second campaign and also previously by Grodent et al. [2003a] and Nichols et al. [2007] using the 2000-2001 Cassini flyby data set. We should note that we discuss the poleward dusk arcs observed during these intervals as if they are independent of the main oval. Thus, for example, in Figure $4 \mathrm{~g}$, the bright poleward arc was considered to be independent of the main oval, which was dim in this sector. However, it may be that this feature is actually the main oval that has moved significantly poleward in this sector at this time, such that the correlation is to be expected. Similarly, the expanded main oval appears to merge with the poleward forms in, e.g., Figures $4 \mathrm{~b}$ and $4 \mathrm{e}$. It thus appears that the bright poleward arcs are at least associated with enhancements in the main oval, if not representing expanded or moved main oval emission. Similarly, the bright, patchy emission that occurs in the low-latitude region during the second campaign may be formed by a process independent of the main oval, or it may represent main oval emission that occurs equatorward of the reference location. We note, however, that sometimes this emission occurs simultaneously with "nominal" main oval auroras, e.g., in Figure 10f. As mentioned in section 3.1, this emission presumably maps to between Io's orbit and $\sim 20 \mathrm{R}_{\mathrm{J}}$, but the question of why it occurred prominently during the second campaign but not the first is presently unanswered.

[43] Although there is some timing uncertainty in the MHD propagation, it, along with the Cassini and New Horizons data indicate that each observation of this particular auroral state can be associated with a solar wind compression region. Compression regions are characterized by overall high dynamic pressure and field strength, but there is typically much variation of these parameters within the compression. The terrestrial aurora has been observed to brighten in response to solar wind shocks [e.g., Boudouridis et al., 2003; Tsurutani and Zhou, 2003], and it is worth 


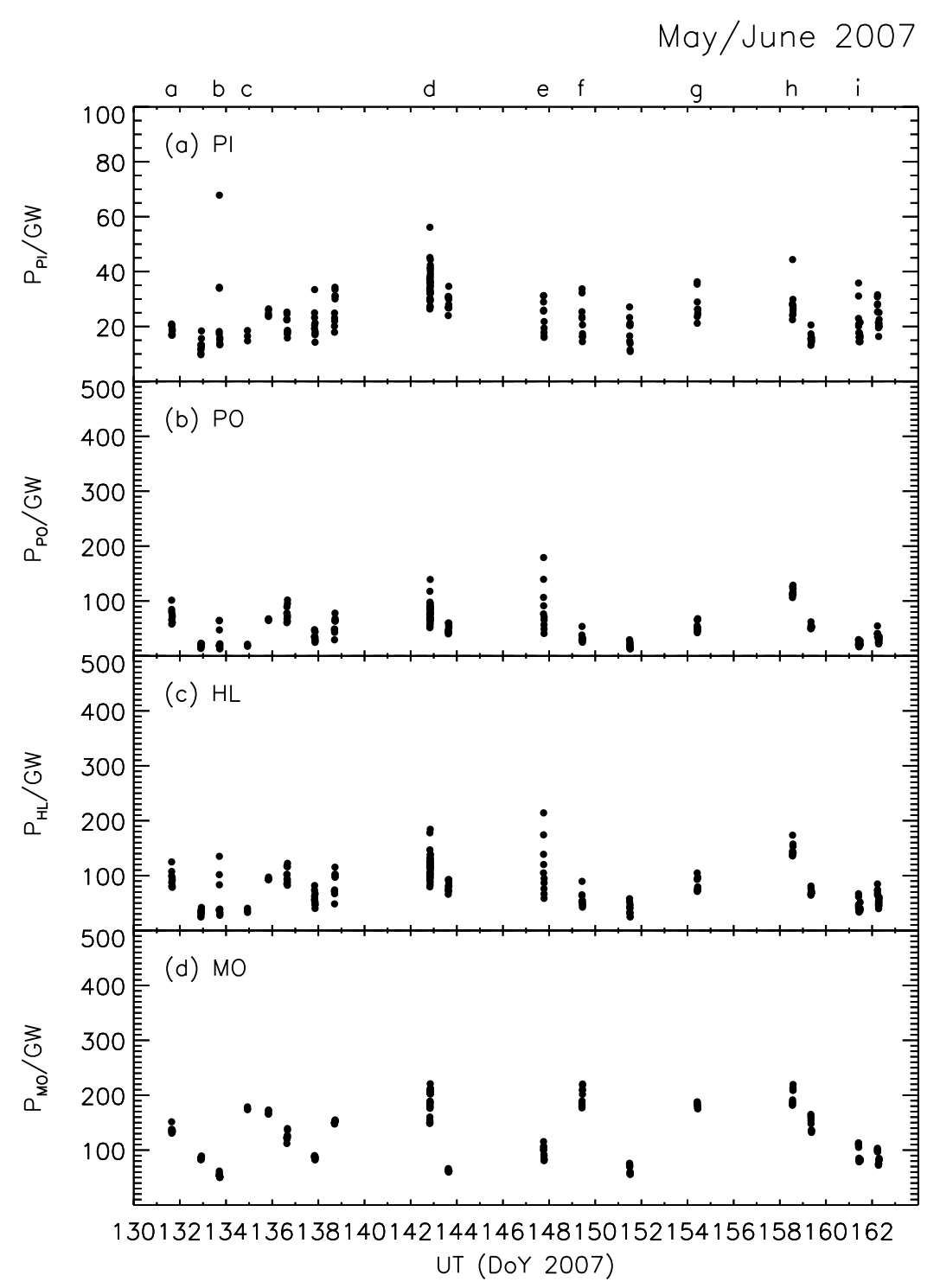

Figure 12. As for Figure 7, but for May-June 2007. Note again the expanded vertical scale in Figure 12a.

discussing here the theoretical expectations of the behavior of the Jovian main oval emission under varying solar wind conditions. The first-order prediction is that the brightness of the main auroral oval should be anticorrelated with the solar wind dynamic pressure, since under magnetospheric compression the angular velocity of the equatorial plasma increases because of conservation of angular momentum, and the corresponding magnetosphere-ionosphere coupling currents will then be decreased, and vice versa. However, there are important caveats to this simple picture. Gong [2005] and Cowley et al. [2007] pointed out that the magnitude of the field-aligned current is also dependent on the timescale of the response of the ionospheric neutrals relative to the ions. This arises since the magnitude of the field-aligned currents is determined by the divergence of the velocity of the ions relative to the neutrals in the Pedersen layer of the planet's ionosphere. If the neutrals are allowed to respond on a timescale similar to the magnetosphereionosphere coupling then the divergence, and therefore field-aligned current, is reduced. If the neutrals are unresponsive on this timescale, however, then the field-aligned current is enhanced. In particular, Cowley et al. [2007] found that for a few hours following an impulsive compression the main oval will dim because of the increased angular velocity of the equatorial plasma, and the emission at the open-closed field line boundary will brighten because of the increased flow shear across the boundary. If the compression is strong enough to induce superrotation as observed by Hanlon et al. [2004], a condition that is achieved by more modest compressions if the neutral atmosphere is unresponsive, then the current system will reverse and instead produce emission on field lines mapping to the outer magnetosphere between the middle magnetosphere and magnetopause. After a few hours the system will revert to the steady state and the usual sense of the current system will be restored, with smaller currents and thus dimmer main oval auroras. 

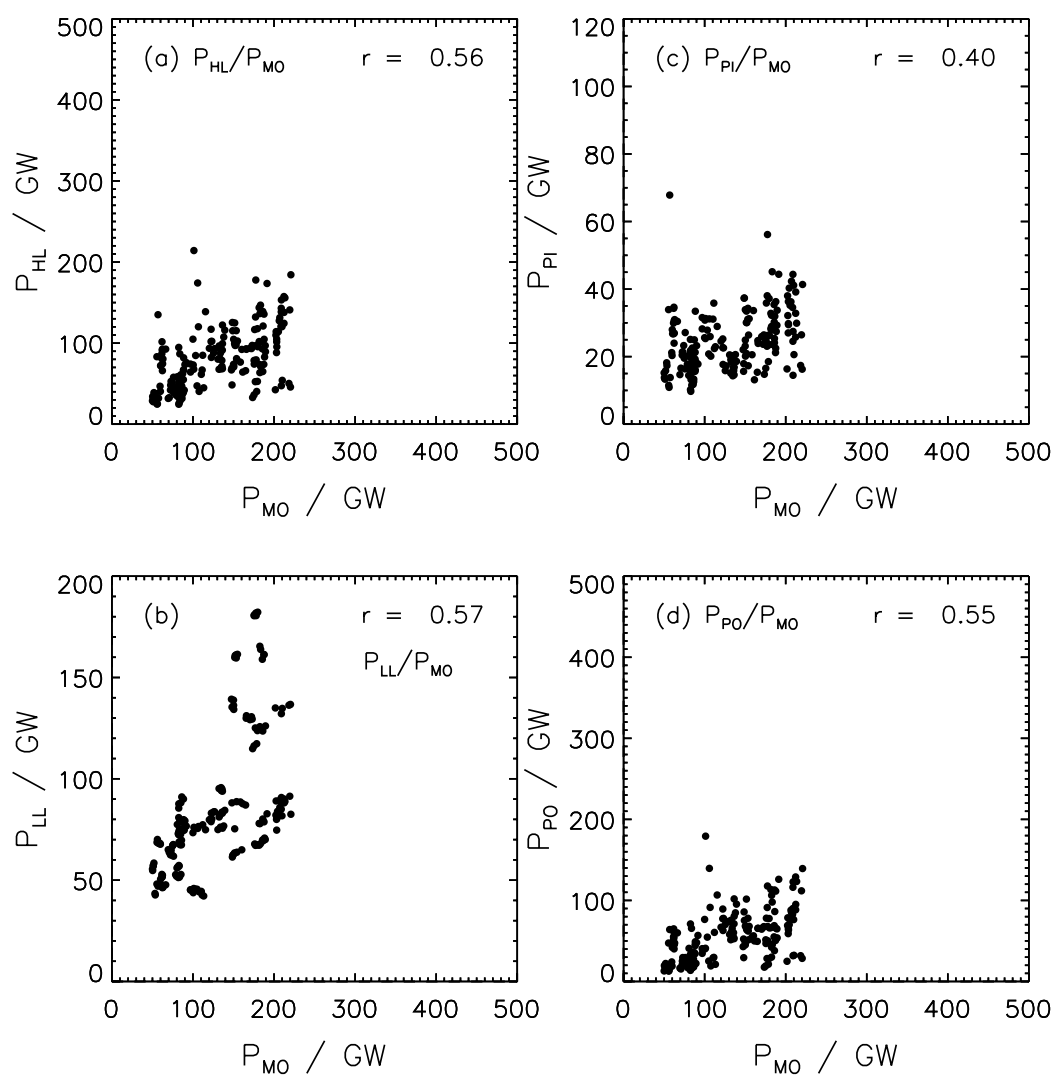

Figure 13. As for Figure 8, but for May-June 2007. Note again the panels have different vertical scales.

[44] We thus note that the morphology over longitudes less than $\sim 180^{\circ}$ described in point 6 in section 6 is not inconsistent with this expectation; that is, the main oval region is dim, and contiguous emission poleward of the previous location of the main oval merges with a poleward bright arc, which would then represent the open closed field line boundary. However, the questions then arise as to why this state lasts up to $\sim 2$ days, rather than the few hours predicted by the theory, and why it is confined to longitudes less than $\sim 180^{\circ}$. It may be that this state is not maintained over $\sim 2$ days, happens a few times per compression region because of the turbulence therein, and merely appears to last this long because of undersampling of HST data. This would explain then why this state is not observed in the first two compression regions in the second campaign. Previous observations obtained during the Cassini flyby [Gurnett et al., 2002; Pryor et al., 2005; Nichols et al., 2007] showed that Jupiter's emitted radio power appeared to be spiky but generally elevated over the full length of the few day long compression regions, whereas the UV power appeared to exhibit isolated spikes in emission lasting 1 Jovian rotation as shown by, e.g., Nichols et al. [2007, Figure 1], although we note that the UV power was significantly undersampled and the exact relation between outer planet radio and UV power is not well understood. With respect to the apparent localization of this effect, we first note that the theories discussed above assume axisymmetry and thus make no statements on this issue. Second, we note that, given the limited CML range of images used in this study, it is possible that the confinement is actually one of local time; that is, the magnetospheric response to compressions is translated into auroral emission differently in the postnoon and prenoon sectors. This local timelongitude ambiguity is an interesting topic that is currently being studied and will be addressed in future works. In any case, this is a particular morphology that is apparently repeatable and is associated with compressions. On the other hand there was no striking phenomenon particularly associated with rarefaction regions or reverse shocks, although we note that in general the reverse shocks were typically far less precipitous than the forward shocks.

[45] With regard to the polar auroras, there were no events which corresponded with the spikes in the field magnitude within the compression regions, but these were rather short lived, and given the uncertainty in the propagation timing and without knowledge of $B_{\mathrm{N}}$ it is impossible to say whether these spikes were in fact effective at causing dayside reconnection. It is not unreasonable to expect that any auroras specifically associated with dayside reconnection, e.g., those discussed by Bunce et al. [2004], may not occur in phase with emission modulated by the solar wind dynamic pressure, since, as indicated in Figures $3 \mathrm{e}$ and 9e, the IMF magnitude is generally largest in the center of compression regions, and the reversing of the IMF at the sector boundaries occurs somewhere within, possibly a few days after the initial shock. Second, very high latitude flux 

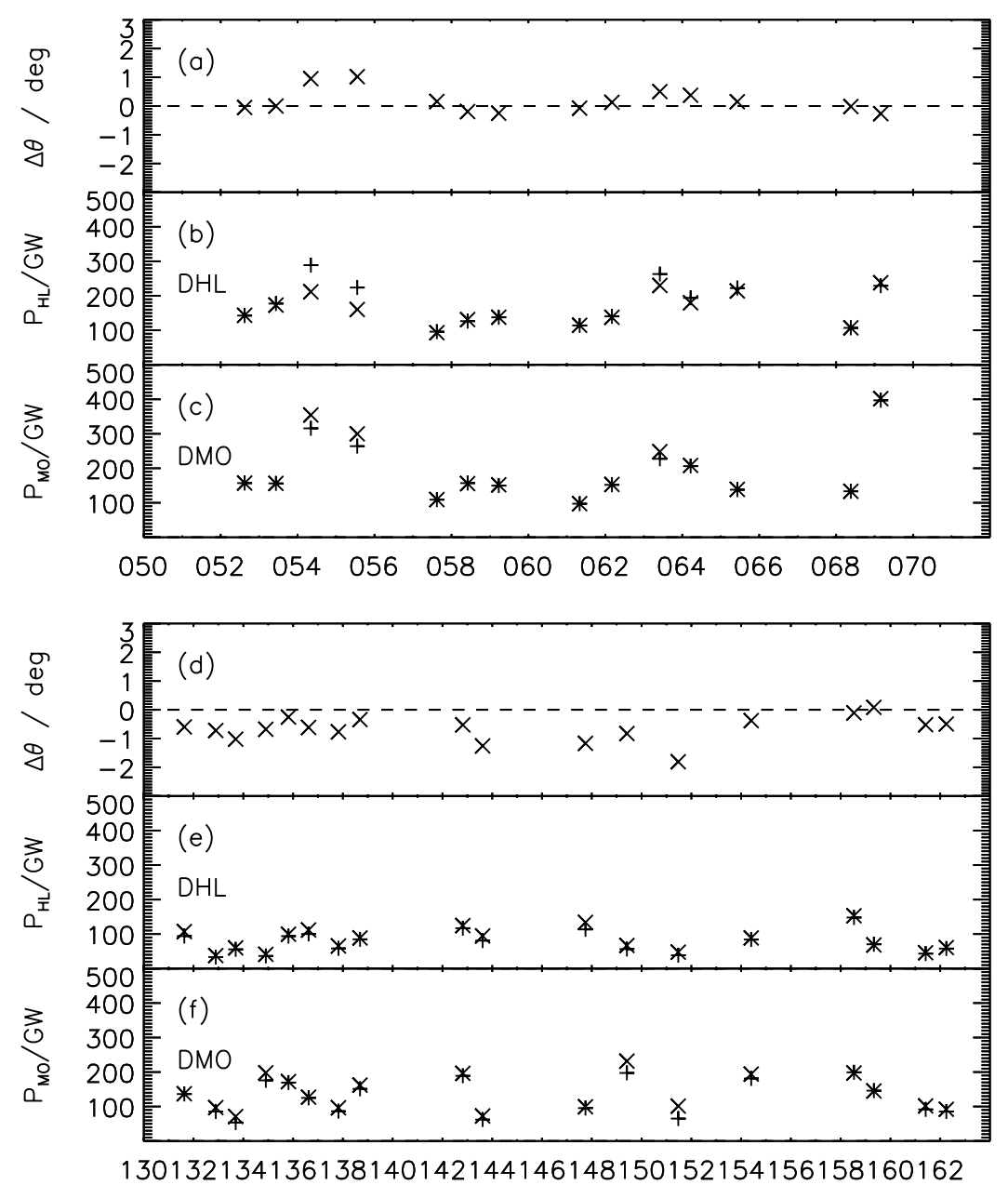

Figure 14. Results obtained using the dynamic definition of the main oval as described in section 3.1. $(\mathrm{a}-\mathrm{c})$ Results for February-March. $(\mathrm{d}-\mathrm{f})$ Results for May-June. In Figures 14a and 14d we show the mean deviations $\Delta \theta$ of the main oval from the fixed reference oval, where positive deviations are toward the center of the oval. The crosses in Figures $14 \mathrm{~b}$ and 14e (labeled DHL) show the daily average of the power emitted from the high-latitude region inside the dynamic main oval, while the pluses show the original values using the fixed main oval. Similarly, Figures $14 \mathrm{c}$ and $14 \mathrm{f}$ (labeled DMO) show the average powers emitted from the dynamic and fixed main oval regions.

tubes may map some distance down the tail, and, e.g., compression induced reconnection in the tail may occur a few days after the shock impinged on the dayside, such if any effects of these reach the planet at all they would likely not coincide with the initial dayside response.

[46] The dawn storm events, characterized by very bright and expanded emission on the dawnside of the main oval, occurred during periods when the estimated solar wind conditions were both compressed (days 69 and 149) and rarefied (day 142). Even allowing for the uncertainties in the MHD model timing, there is no immediately apparent solar wind trigger for these events. Interestingly, we have shown that the brightening is not confined to merely the main oval, the power emitted from the high-latitude auroras increased dramatically too, at least on days 69 and 142. This brightening cannot be explained by expanded main oval auroras contaminating the high-latitude region, since the polar inner auroras, defined as being at least $7^{\circ}$ away from the reference main oval, also increased in emitted power. The dawn storm phenomenon thus affects flux tubes that map to very different regions of the magnetosphere, from the middle magnetosphere to the open field region, and the cause is, as yet, unknown.

[47] Acknowledgments. This work is based on observations made with the NASA/ESA Hubble Space Telescope, obtained at the Space Telescope Science Institute, which is operated by AURA, Incorporated, for NASA. Work at Boston was supported during the course of this study by NASA grant HST-GO-10862.01-A from the Space Telescope Science Institute to Boston University. Work at Leicester was supported by STFC grant PP/E000983/1. J.C.G. and D.G. are supported by the Belgian Fund for Scientific Research (FNRS) and the PRODEX Programme managed by the European Space Agency in collaboration with the Belgian Federal Science Policy Office. J.D.N. wishes to thank S.W.H. Cowley for useful comments on the manuscript.

[48] Wolfgang Baumjohann thanks Wayne Pryor and another reviewer for their assistance in evaluating this paper.

\section{References}

Acton, C. H. (1996), Ancillary data services of NASA's Navigation and Ancillary Information Facility, Planet. Space Sci., 44, 65-70. 
Ballester, G. E., et al. (1996), Time-resolved observations of Jupiter's farultraviolet aurora, Science, 274, 409-412.

Bonfond, B., D. Grodent, J.-C. Gérard, A. Radioti, J. Saur, and S. Jacobsen (2008), UV Io footprint leading spot: A key feature for understanding the UV Io footprint multiplicity?, Geophys. Res. Lett., 35, L05107, doi:10.1029/2007GL032418.

Boudouridis, A., E. Zesta, L. R. Lyons, P. C. Anderson, and D. Lummerzheim (2003), Effect of solar wind pressure pulses on the size and strength of the auroral oval, J. Geophys. Res., 108(A4), 8012, doi:10.1029/ 2002JA009373.

Bunce, E. J., S. W. H. Cowley, and T. K. Yeoman (2004), Jovian cusp processes: Implications for the polar aurora, J. Geophys. Res., 109 A09S13, doi:10.1029/2003JA010280.

Clarke, J. T., H. W. Moos, S. K. Atreya, and A. L. Lane (1980), Observations from earth orbit and variability of the polar aurora on Jupiter, Astrophys. J., 241, L179-L182, doi:10.1086/183386.

Clarke, J. T., L. Ben Jaffel, and J.-C. Gérard (1998), Hubble Space Telescope imaging of Jupiter's UV aurora during the Galileo orbiter mission, J. Geophys. Res., 103, 20,217-20,236, doi:10.1029/98JE01130.

Clarke, J. T., et al. (2002), Ultraviolet emissions from the magnetic footprints of Io, Ganymede and Europa on Jupiter, Nature, 415, 997-1000.

Clarke, J. T., D. Grodent, S. W. H. Cowley, E. J. Bunce, P. Zarka, J. E. P. Connerney, and T. Satoh (2004), Jupiter's aurora, in Jupiter. The Planet, Satellites and Magnetosphere, edited by F. Bagenal, T. E. Dowling, and W. B. McKinnon, pp. 639-670, Cambridge. Univ. Press, Cambridge, U. K.

Clarke, J. T., et al. (2009), The response of Jupiter's and Saturn's auroral activity to the solar wind, J. Geophys. Res., 114, A05210, doi:10.1029/ 2008JA013694.

Cowley, S. W. H., and E. J. Bunce (2001), Origin of the main auroral oval in Jupiter's coupled magnetosphere-ionosphere system, Planet. Space Sci., 49, 1067-1088.

Cowley, S. W. H., and E. J. Bunce (2003a), Modulation of Jupiter's main auroral oval emissions by solar wind induced expansions and compressions of the magnetosphere, Planet. Space Sci., 51, 57-79.

Cowley, S. W. H., and E. J. Bunce (2003b), Modulation of Jovian middle magnetosphere currents and auroral precipitation by solar wind-induced compressions and expansions of the magnetosphere: Initial response and steady state, Planet. Space Sci., 51, 31-56.

Cowley, S. W. H., I. I. Alexeev, E. S. Belenkaya, E. J. Bunce, C. E. Cottis, V. V. Kalegaev, J. D. Nichols, R. Prangé, and F. J. Wilson (2005), A simple axisymmetric model of magnetosphere-ionosphere coupling currents in Jupiter's polar ionosphere, J. Geophys. Res., 110, A11209, doi:10.1029/2005JA011237.

Cowley, S. W. H., J. D. Nichols, and D. J. Andrews (2007), Modulation of Jupiter's plasma flow, polar currents, and auroral precipitation by solar wind-induced compressions and expansions of the magnetosphere: a simple theoretical model, Ann. Geophys., 25, 1433-1463.

Elliott, H. A. (2007), New Horizons SWAP solar wind measurements at Jupiter encounter, paper presented at the Magnetospheres of the Outer Planets Conference, Southwest Res. Inst., San Antonio, Tex., 25-29 June.

Elsner, R. F., et al. (2005), Simultaneous Chandra X ray, Hubble Space Telescope ultraviolet, and Ulysses radio observations of Jupiter's aurora, J. Geophys. Res., 110, A01207, doi:10.1029/2004JA010717.

Gérard, J.-C., J. Gustin, D. Grodent, P. Delamere, and J. T. Clarke (2002), Excitation of the FUV Io tail on Jupiter: Characterization of the electron precipitation, J. Geophys. Res., 107(A11), 1394, doi:10.1029/ 2002JA009410.

Gérard, J.-C., A. Saglam, D. Grodent, and J. T. Clarke (2006), Morphology of the ultraviolet Io footprint emission and its control by Io's location, J. Geophys. Res., 111, A04202, doi:10.1029/2005JA011327.

Gong, B. (2005), Variations of Jovian aurora induced by changes in solar wind dynamic pressure, Ph.D. thesis, Rice Univ., Houston, Tex.

Grodent, D., J. T. Clarke, J. Kim, J. H. Waite, and S. W. H. Cowley (2003a), Jupiter's main auroral oval observed with HST-STIS, J. Geophys. Res., 108(A11), 1389, doi:10.1029/2003JA009921.

Grodent, D., J. T. Clarke, J. H. Waite, S. W. H. Cowley, J.-C. Gérard, and J. Kim (2003b), Jupiter's polar auroral emissions, J. Geophys. Res. 108(A10), 1366, doi:10.1029/2003JA010017.

Grodent, D., B. Bonfond, J.-C. Gérard, A. Radioti, J. Gustin, J. T. Clarke, J. Nichols, and J. E. P. Connerney (2008a), Auroral evidence of a localized magnetic anomaly in Jupiter's northern hemisphere, J. Geophys. Res., 113, A09201, doi:10.1029/2008JA013185.
Grodent, D., J.-C. Gérard, A. Radioti, B. Bonfond, and A. Saglam (2008b), Jupiter's changing auroral location, J. Geophys. Res., 113, A01206, doi:10.1029/2007JA012601.

Gurnett, D. A., et al. (2002), Control of Jupiter's radio emission and aurorae by the solar wind, Nature, 415, 985-987.

Gustin, J., S. W. H. Cowley, J.-C. Gérard, G. R. Gladstone, D. Grodent, and J. T. Clarke (2006), Characteristics of Jovian morning bright FUV aurora from Hubble Space Telescope/Space Telescope Imaging Spectrograph imaging and spectral observations, J. Geophys. Res., 111, A09220, doi:10.1029/2006JA011730.

Hanlon, P. G., M. K. Dougherty, N. Krupp, K. C. Hansen, F. J. Crary, D. T. Young, and G. Tóth (2004), Dual spacecraft observations of a compression event within the Jovian magnetosphere: Signatures of externally triggered supercorotation?, J. Geophys. Res., 109, A09S09, doi:10.1029/2003JA010116.

Hill, T. W. (2001), The Jovian auroral oval, J. Geophys. Res., 106, $8101-$ 8108, doi:10.1029/2000JA000302.

Khurana, K. K. (2001), Influence of solar wind on Jupiter's magnetosphere deduced from currents in the equatorial plane, J. Geophys. Res., 106 , 25,999-26,016, doi:10.1029/2000JA000352.

Nichols, J. D., and S. W. H. Cowley (2004), Magnetosphere-ionosphere coupling currents in Jupiter's middle magnetosphere: Effect of precipitation-induced enhancement of the ionospheric Pedersen conductivity, Ann Geophys., 22, 1799-1827.

Nichols, J. D., S. W. H. Cowley, and D. J. McComas (2006), Magnetopause reconnection rate estimates for Jupiter's magnetosphere based on interplanetary measurements at 5AU, Ann. Geophys., 24, 393-406.

Nichols, J. D., E. J. Bunce, J. T. Clarke, S. W. H. Cowley, J-.C. Gérard, D. Grodent, and W. R. Pryor (2007), Response of Jupiter's UV auroras to interplanetary conditions as observed by the Hubble Space Telescope during the Cassini flyby campaign, J. Geophys. Res., 112, A02203, doi:10.1029/2006JA012005.

Pallier, L., and R. Prangé (2001), More about the structure of the high latitude Jovian aurorae, Planet. Space Sci., 49, 1159-1173.

Prangé, R., G. Chagnon, M. G. Kivelson, T. A. Livengood, and W. Kurth (2001), Temporal monitoring of Jupiter's auroral activity with IUE during the Galileo mission. Implications for magnetospheric processes, Planet. Space Sci. 49, 405-415.

Pryor, W. R., et al. (2005), Cassini UVIS observations of Jupiter's auroral variability, Icarus, 178, 312-326, doi:10.1016/j.icarus.2005.05.021.

Radioti, A., D. Grodent, J.-C. Gérard, B. Bonfond, and J. T. Clarke (2008), Auroral polar dawn spots: Signatures of internally driven reconnection processes at Jupiter's magnetotail, Geophys. Res. Lett., 35, L03104, doi:10.1029/2007GL032460.

Serio, A. W., and J. T. Clarke (2008), The variation of Io's auroral footprint brightness with the location of Io in the plasma torus, Icarus, 197, $368-$ 374, doi:10.1016/j.icarus.2008.03.026.

Southwood, D. J., and M. G. Kivelson (2001), A new perspective concerning the influence of the solar wind on the Jovian magnetosphere, J. Geophys. Res., 106, 6123-6130, doi:10.1029/2000JA000236.

Tsurutani, B. T., and X. Y. Zhou (2003), Interplanetary shock triggering of substorms: Wind and polar, Adv. Space Res., 31(4), 1063-1067, doi:10.1016/S0273-1177(02)00796-2.

Vasavada, A. R., A. H. Bouchez, A. P. Ingersoll, B. Little, C. D. Anger, and the Galileo SSI team (1999), Jupiter's visible aurora and Io footprint, J. Geophys. Res., 104, 27,133-27,142.

Vincent, M. B., et al. (2000), Mapping Jupiter's latitudinal bands and Great Red Spot using HST/WFPC2 far-ultraviolet imaging, Icarus, 143, 189204, doi:10.1006/icar.1999.6232

Waite, J. H., et al. (2001), An auroral flare at Jupiter, Nature, 410, 787 789.

Zieger, B., and K. C. Hansen (2008), Statistical validation of a solar wind propagation model from 1 to 10 AU, J. Geophys. Res., 113, A08107, doi:10.1029/2008JA013046.

J. Clarke, Center for Space Physics, Boston University, 725 Commonwealth Avenue, Boston, MA 02215, USA.

J. C. Gérard and D. Grodent, Laboratory for Planetary and Atmospheric Physics, Institut d'Astrophysique et de Géophysique, Université de Liège, B5c Allée du 6 aout, 17, B-4000 Liège, Belgium.

K. Hansen, Department of Atmospheric, Oceanic and Space Sciences, University of Michigan, 2455 Hayward Street, Ann Arbor, MI 48109-2143, USA.

J. D. Nichols, Department of Physics and Astronomy, University of Leicester, Leicester LE1 7RH, UK. (jdn@ion.le.ac.uk) 